\title{
An Instance Segmentation and Clustering Model for Energy Audit Assessments in Built Environments: A Multi-Stage Approach
}

\author{
Youness Arjoune ${ }^{\dagger} \ddagger\left(\mathbb{D}\right.$, Sai Peri $\ddagger$, Niroop Sugunaraj $*,+\neq \oplus$, Avhishek Biswas ${ }^{\dagger}$, Debanjan Sadhukhan $\ddagger$ \\ and Prakash Ranganathan $t \neq$
}

check for

updates

Citation: Arjoune, Y.; Peri, S.;

Sugunaraj, N.; Biswas, A.;

Sadhukhan, D.; Ranganathan, P. An

Instance Segmentation and Clustering

Model for Energy Audit Assessments

in Built Environments: A Multi-Stage

Approach. Sensors 2021, 21, 4375.

https://doi.org/10.3390/s21134375

Academic Editors: Sukho Lee, Dae-Ki Kang, Gian Marco Revel and Sylvain Girard

Received: 25 March 2021

Accepted: 21 June 2021

Published: 26 June 2021

Publisher's Note: MDPI stays neutral with regard to jurisdictional claims in published maps and institutional affiliations.

Copyright: (c) 2021 by the authors. Licensee MDPI, Basel, Switzerland. This article is an open access article distributed under the terms and conditions of the Creative Commons Attribution (CC BY) license (https:// creativecommons.org/licenses/by/ $4.0 /)$.
School of Electrical Engineering and Computer Science (SEECS), University of North Dakota (UND), Grand Forks, ND 58201, USA; youness.arjoune@und.edu (Y.A.); sai.peri@und.edu (S.P.); avhishek.biswas@und.edu (A.B.); debanjan.sadhukhan1987@gmail.com (D.S.); prakash.ranganathan@und.edu (P.R.)

* Correspondence: niroop.sugunaraj@und.edu

+ Current address: 243 Centennial Dr Stop 8155, Grand Forks, ND 58201, USA.

$\ddagger$ These authors contributed equally to this work.

\begin{abstract}
Heat loss quantification (HLQ) is an essential step in improving a building's thermal performance and optimizing its energy usage. While this problem is well-studied in the literature, most of the existing studies are either qualitative or minimally driven quantitative studies that rely on localized building envelope points and are, thus, not suitable for automated solutions in energy audit applications. This research work is an attempt to fill this gap of knowledge by utilizing intensive thermal data (on the order of 100,000 plus images) and constitutes a relatively new area of analysis in energy audit applications. Specifically, we demonstrate a novel process using deep-learning methods to segment more than 100,000 thermal images collected from an unmanned aerial system (UAS). To quantify the heat loss for a building envelope, multiple stages of computations need to be performed: object detection (using Mask-RCNN/Faster R-CNN), estimating the surface temperature (using two clustering methods), and finally calculating the overall heat transfer coefficient (e.g., the U-value). The proposed model was applied to eleven academic campuses across the state of North Dakota. The preliminary findings indicate that Mask R-CNN outperformed other instance segmentation models with an $\mathrm{mIOU}$ of $73 \%$ for facades, $55 \%$ for windows, $67 \%$ for roofs, $24 \%$ for doors, and $11 \%$ for HVACs. Two clustering methods, namely K-means and threshold-based clustering (TBC), were deployed to estimate surface temperatures with TBC providing consistent estimates across all times of the day over K-means. Our analysis demonstrated that thermal efficiency not only depended on the accurate acquisition of thermal images but also relied on other factors, such as the building geometry and seasonal weather parameters, such as the outside/inside building temperatures, wind, time of day, and indoor heating/cooling conditions. Finally, the resultant U-values of various building envelopes were compared with recommendations from the American Society of Heating, Refrigerating, and Air-conditioning Engineers (ASHRAE) building standards.
\end{abstract}

Keywords: clustering; heat loss quantification; instance segmentation; Mask R-CNN; deep learning; mean average precision; thermal imagery; UASs; U-value; ASHRAE

\section{Introduction}

Heat loss quantification (HLQ) plays a crucial role in reducing the overall energy consumption for optimal operations in buildings, particularly since its usage has a considerable impact on the environment and a building's life cycle [1,2]. A key objective of HLQ is retrofitting existing building envelopes. The first necessary step in the building envelope optimization process is assessing the actual thermal performance. Indicators, such as the energy performance or energy use intensity, are used to express this performance. 
The building envelope in-situ measurement depends on factors that are classified into three main categories: site conditions, building conditions, and operating conditions [3]. The site conditions category refers to the weather conditions under which the tests are performed. These weather conditions include, but are not limited to, wind velocity, rain, solar radiation, and humidity, all of which can significantly alter the building's thermal performance quantification.

The building condition category refers to the age of the building materials and the laying of the structural elements used during construction. The operating conditions category refers to the building's environmental management, such as heating or cooling, and air circulation from the opening or closing of windows, and building maintenance, regardless of whether or not these activities are currently affecting the building envelope. All of these factors must be monitored and considered carefully during the evaluation of building heat loss quantification.

Multiple research groups have recently investigated the use of infrared thermography to measure building envelope parameters in-situ with the thermal transmittance values, or the amount of heat-flow in one square meter when the temperature difference is one Kelvin (U-value). Most of these techniques present unique challenges [4]. The in-situ-based measurement of the U-value, along with the heat flowmeter method (HFM), is not always possible [5] or accurate [6] due to the assumptions upon which the HFM methods are based, such as uni-directional heat flow; therefore, it is of crucial importance to develop practical techniques that quantify the heat loss.

One technique to obtain thermal imagery is by using drones, which creates opportunities in building assessment and inspections [7-9]. Drones enables fast and safe building inspections, which are necessary to complete proactive maintenance to mitigate problems before they become costly. Reducing the costs associated with insurance inspections is another benefit to building owners and managers. The risks associated with using drones for roof inspection are low compared to traditional methods, where employees risk injury as they traverse the building to inspect the structure. Drones are increasingly used for data collection; however, thermal images captured by drones often contain objects, such as trees and ground surfaces, all of which can impact the calculation of the U-value calculation [10]. Instance segmentation of the regions of interest, such as the facade of the building or roofs, is a necessary step after collecting data.

Over the past two decades, several instance segmentation and masking techniques have been considered using machine learning-based methods, which are the most successful at identifying objects [11,12]. Machine learning applies complex mathematical models to uncover hidden correlations between the different features in a given data set. There are two types of machine learning techniques: supervised and unsupervised. Supervised techniques require specific rules that an expert provides for the machine. These rules allow the machine to either classify or predict the outputs of the model given an input. Unsupervised techniques are applied when an expert cannot provide rules because of the large data sets. These techniques are applied to reveal any hidden correlations that the expert may not notice. Machine learning techniques require feature selection, which requires human expertise to determine appropriate features. A recent class of machine learning, called deep learning, does not require this step and is a new and powerful technique for computer vision tasks, which has not fully exploited by the heat loss research community $[13,14]$.

In addition, the current research in heat loss quantification has relied largely on qualitative bench-top solutions or localized analysis in energy audit building evaluation. The existing quantitative models used to estimate surface temperature in buildings do not account for multi-variate uncertainties, such as energy requirement/consumption patterns, multiple sets of images per building object, time-of-day, seasons, and building material. There are also inconsistencies in the process of arriving at a reliable and quantifiable Uvalues for a building envelope. 
For example, the thermal readings taken from a combination of thermocouple sensors and a thermal camera are only "raw values" that need to be pre-processed and subjected to multiple uncertainties (e.g., solar radiance, wind speed, time of the day, and black body radiation) and, thus, require the need for post-processing stages. In addition, the current literature does not provide guidance on how to arrive at an optimal and accurate way to detect building objects through which one can estimate surface temperatures from the region of interest (ROI) through heat loss metrics. Existing solutions are, therefore, not reliable for energy audit applications.

The specific research questions our work addresses include:

1. How do we acquire and process thermal images that account for building geometry uncertainties, such as orientation and angle, seasonal changes, and the influence of weather parameters on the building envelope?

2. How do we accurately detect various instances of objects, such as walls, roofs, and windows, using data-driven approaches?

3. How do we automatically tag or label images and report them?

Our work covers a comprehensive data-driven approach that examined approximately 100,000 thermal images, and performed object identification to classify objects accurately using instance segmentation to detect various building envelope structures, such roofs, walls, doors, windows, and facades. We also created a method for automated tagging, tracked pixel-pixel surface temperature values and reported the values in quantifiable and standard U-value estimation units.

There exists hundreds of variations of U-value formulae in the existing literature, and often they do not use large data but instead single point values. We investigated all available $U$-value equations and developed a cumulative $U$-value formula from three existing U-value equations. The U-value is a series of heat transfer equations that account for pixel temperature, outside temperature, wind speed, etc., to develop a quantitative measure of how that particular object is performing compared to how it should theoretically be performing. It can be thought of as an extension to the direct thermal readings, but we account for it from multiple low ceiling thermal images per building object acquired from small scale aerial systems.

We deployed two clustering methods (e.g., K-means and threshold-based clustering), which were then developed to estimate the accurate surface temperatures of multiple instances of an object. Then, using the estimated surface temperature for the region or envelope, we developed a cumulative U-value $\left(U_{c}\right)$ formula that uses multiple existing U-value equations from the literature. We empirically verified our $U_{c}$ as the most accurate formula when using a benchmark to meet the ASHRAE standard recommendations. $U_{c}$ demonstrated relatively lower errors compared to the other U-value equations. The statistical difference of the U-value building envelope computations against ASHRAE varied between 0 to $30 \%$ depending on area size, building type, and material used. Since our AI model can detect multiple instances of any object with greater accuracy, including the roof, windows, doors, HVACs, and facades, the model is unique, it fills the research gap of inaccuracies and provides a quantifiable way to address uncertainties.

Our work adds to the body of knowledge by addressing the lack of automated solutions in energy audit applications and providing a comprehensive view of the building envelopes that will result in reliable, quantifiable, and scalable workflows to address heat loss quantification problems for next-generation building inspection problems. We determined that the thermal efficiency of a building depends on multiple factors, not only on the accurate acquisition of thermal images, but on factors such as the building geometry, season of the year, time of day, indoor heating or cooling conditions, past historical consumption, and power generation sources. These factors are all influential in determining the overall assessment of an energy audit evaluation.

In this paper, we demonstrated that thermal imagery to quantify heat loss combined with the recent advances in deep learning theory has many advantages, such as remote sensing, flexibility, and minimizing injury risks. To the best of our knowledge, the pro- 
posed approach is the first of its kind. U-values for different building blocks were analyzed and compared to the American Society of Heating, Refrigerating, and Air-conditioning Engineers (ASHRAE) building standards. This approach will allow stakeholders to overcome the challenges of traditional heat loss quantification methods. This work expounds upon previous publications $[4,15]$ with the following new subject material,

- A large thermal image data repository ( 100,000 images) of multiple university buildings was collected using a UAS and manually annotated to highlight objects of interest, such as facades, walls, trees, roofs, and windows.

- Multiple models for object detection, such as Mask R-CNN, Fast R-CNN, and Faster R-CNN, were trained on several backbone types and validated with metrics, such as average precision (AP) and intersection-over-union (IoU), through a data-driven three-layered framework.

- Two clustering schemes were tested to estimate surface temperature readings and identify hotspot regions reliably. Quantified surface temperature observations were used to compute the U-values of objects and validated with the ASHRAE standards.

The relationship between the indoors and HLQ is essential; however, this is out of the scope of this paper since our main focus is to provide a heat loss estimation using thermal imagery of the buildings from the outside and from which the heat loss is determined.

The rest of this paper is organized as follows: Section 2 presents state-of-the-art techniques for object detection and instance segmentation. We devoted a specific section for this and did not integrate it as subsection of the introduction so as to not interrupt the flow of the paper as this section provides an in-depth review of the recent advances in deep learning and computer vision. This section provides also useful knowledge that can help in developing novel computer vision techniques. Section 3 describes the methodology used in this paper to quantify the heat loss. We describe the training and testing methodology of several computer vision techniques as well as the analytical formulas used to calculate U-values through a three-layered framework. Section 3 also describes the clustering techniques applied to detect hotspot regions within thermal images. Section 4 presents the evaluation metrics and examples of the obtained results, which includes the results of instance segmentation, clustering analyses, heat loss quantification using U-values, and a qualitative and quantitative uncertainty analysis. Section 5 summarizes the paper, presents our conclusions, and suggests future research work.

\section{Related Work on Computer Vision and Image Clustering}

In this section, we provide an overview of the recent advances in computer vision and the state-of-the-art algorithms for object detection and segmentation. This section provides also some related work on image clustering and the detection of hotspots.

\subsection{Object Detection and Instance Segmentation: The State of the Art}

Computer vision can be classified into either object detection or segmentation. Object detection is more specific than classification in that it must draw a bounding box (BB) around every object identified [16]. If an object detected has been identified completely, including all pixels, it is considered a segmentation. Segmentation methods can be further divided into semantic, instance, and panoptic. All pixels belonging to all objects of the same class are classified as one image segment in semantic segmentation. Instance segmentation classifies each instance as a segment even if the image is formed of objects of the same class.

Panoptic segmentation combines both instance segmentation and semantic segmentation by assigning class labels to each unique object segmentation. Object detection and segmentation has been performed in the past by using traditional techniques, such as histogram gradients. Deep learning in computer vision is gaining popularity, as it has been recognized as an effective technique compared to traditional methods [17]. Table 1 summarizes the popular machine learning models used to perform computer vision tasks, with a brief discussion on their performances. 
Table 1. State-of-the-art computer vision techniques.

\begin{tabular}{|c|c|c|c|c|}
\hline Computer Vision Task & Model & Structure/Backbone & Metrics & Comments \\
\hline \multirow{4}{*}{ Detection } & R-CNN $[18,19]$ & $\begin{array}{c}\text { Selective Search Algorithm + } \\
\text { SVM }\end{array}$ & $\begin{array}{c}\text { mAP of } 62 \% \text { on PASCAL } \\
\text { VOC } 2012\end{array}$ & $\begin{array}{l}\text { Slow because of the high } \\
\text { number of region proposals } \\
(2000)(47 \mathrm{~s} / \text { test image })\end{array}$ \\
\hline & Fast R-CNN [20] & $\begin{array}{l}\text { Selective Search Algorithm + } \\
\text { FCs }\end{array}$ & $\begin{array}{c}\text { mAP of } 39.3 \% @ 0.5 \text { on MS } \\
\text { COCO and 66\% on PASCAL } \\
\text { VOC } 2012\end{array}$ & $\begin{array}{c}\text { "Fast compared with R-CNN } \\
0.32 \mathrm{~s} / \text { testing image Fast } \\
\text { R-CNN trains the very deep } \\
\text { VGG16 network } 9 \times \text { faster } \\
\text { than R-CNN, is } 213 \times \text { faster } \\
\text { at test-time" }\end{array}$ \\
\hline & Faster R-CNN [21-23] & $\begin{array}{l}\text { Region Proposal Network + } \\
\text { ROI pooling + FCs }\end{array}$ & $\begin{array}{c}\mathrm{mAP} \text { of } 42.7 \% \text { on MS COCO } \\
\text { and } \mathrm{mAP} \text { of } 78.8 \% \text { PASCAL } \\
\text { VOC } 2012\end{array}$ & $\begin{array}{l}\text { Remove Selective Search } \\
\text { Algorithm }\end{array}$ \\
\hline & SSD & ResNet101 + FCs & $\mathrm{mAP}$ of $31.2 \%$ & Runs at $125 \mathrm{~ms}$ \\
\hline Real-Time Object Detection & YOLO [24,25] & $\begin{array}{l}\text { Single Regression from image } \\
\text { pixel to class BB (Darknet-53) }\end{array}$ & $\begin{array}{c}\text { YOLOv3-320 mAP of } 28.2 \%, \\
\text { YOLOv3-416 mAP of } 31 \% \text {, } \\
\text { and YOLOv3-608 mAP of } \\
33 \% "\end{array}$ & $\begin{array}{l}\text { Support real-time (up to } \\
45 \text { FPS for YOLOv3-320) }\end{array}$ \\
\hline \multirow{4}{*}{ Semantic Segmentation } & DeconvNet [26] & $\begin{array}{l}\text { ConvNet (VGG-16) } \\
\text { concatenated with } \\
\text { DeconvNet }\end{array}$ & $\begin{array}{c}\text { mAP of } 70 \% \text { on PASCAL } \\
\text { VOC } 2012\end{array}$ & \multirow{3}{*}{ None } \\
\hline & FCN [27] & $\begin{array}{l}\text { FCN introduces the skip } \\
\text { connection to fuse feature } \\
\text { layers of different scales }\end{array}$ & $\begin{array}{c}\text { Graph-FCN achieves mIoU of } \\
65.91 \% \text { on PASCAL VOC } \\
\text { Dataset and FCN-32 achieves } \\
\text { mIoU of } 36.64 \% \text { on } \\
\text { PASCAL-Context }\end{array}$ & \\
\hline & ParsNet [28] & Improved FCN & $\begin{array}{l}\text { ParseNet Baseline achieves } \\
\text { mIoU of } 67.3 \% \text { on PASCAL } \\
\text { VOC Dataset and ParseNet } \\
\text { achieves mIoU of } 69.8 \% \text { on } \\
\text { PASCAL VOC Dataset }\end{array}$ & \\
\hline & Deeplab [29] & $\begin{array}{l}\text { Atrous Convolution for } \\
\text { Dense Feature Extraction + } \\
\text { Atrous Spatial Pyramid } \\
\text { Pooling + Fully-Connected } \\
\text { Conditional Random Fields }\end{array}$ & $\begin{array}{c}\text { DeepLab achives mIoU of } \\
64.96 \% \text { on PASCAL VOC } \\
2012 \text { and DeepLab-LargeFOV } \\
\text { achieves mIoU of } 65.82 \% \text { on } \\
\text { PASCAL VOC } 2012\end{array}$ & $\begin{array}{l}\text { Objective function is } \\
\text { optimized in all layers with } \\
\text { respect to weights by SGD } \\
\text { standards. Imagenet classifier } \\
\text { is replaced with classifier } \\
\text { equaling number of target } \\
\text { classes in last layer. }\end{array}$ \\
\hline \multirow{2}{*}{ Instance Segmentation } & EncNet [30] & $\begin{array}{c}\text { ResNet }+ \text { Context Encoding } \\
\text { Module }\end{array}$ & $\begin{array}{c}\text { mIoU of } 52.6 \% \text { on } \\
\text { PASCAL-Context Dataset }\end{array}$ & $\begin{array}{l}\text { Introduces very little extra } \\
\text { computation to original FCN } \\
\text { network. Context Encoder is } \\
\text { light weight. }\end{array}$ \\
\hline & Mask R-CNN [20,31,32] & RPN + $\underset{\text { ResNet101 }}{\text { ROIAlign }+ \text { Mask }+}$ & $\begin{array}{c}\text { mAP of } 39.8 \% \text { and } 63.1 \% \text { on } \\
\text { MS COCO for keypoints \& } \\
\text { mask }\end{array}$ & $\begin{array}{l}\text { Replaces ROI pooling with } \\
\text { ROIAlign in Faster R-CNN } \\
\text { architecture and includes } \\
\text { FCN for segmentation }\end{array}$ \\
\hline Panoptic Segmentation & $\begin{array}{l}\text { Machine panoptic } \\
\text { segmentation [33] }\end{array}$ & $\begin{array}{c}\text { Unified semantic } \\
\text { segmentation and instance } \\
\text { segmentation }\end{array}$ & $\begin{array}{c}P Q^{\text {th }} \text { of } 54 \%, 79.4 \%, 67.8 \% \text { on } \\
\text { Cityscape }\end{array}$ & $\begin{array}{l}\text { Unique evaluation } \\
\text { methodology }\end{array}$ \\
\hline
\end{tabular}

Applying a classifier, such as a convolutional neural network (CNN), for detecting the presence of an object within each region of interest by splitting the images is an incomplete approach for deep learning. CNN, concatenated with a classifier, such as fully-connected layers (FCs), cannot be used for the number of object occurrences in each image since the objects are not the same and, thereby, change the length of the output layer. Region-based $\mathrm{CNN}$ (R-CNN) has been proposed to mitigate this issue. This algorithm extracts region proposals with approximately 2000 regions using a selective search algorithm.

These proposals are then used to form warped regions on which a CNN is applied for feature extraction. This latter feature extraction is fed to a support vector machine classifier to classify the regions. Even though R-CNN performs well, it must repeat this process for all images, each of which requires 2000 processed regions. Each test image requires $47 \mathrm{~s}$ to process; therefore, $\mathrm{R}-\mathrm{CNN}$ is infeasible even though it performs well. R-CNN achieves an mAP of $62 \%$ on PASCAL-VOC-2012.

A method utilizing a similar approach to $\mathrm{R}-\mathrm{CNN}$ with some manipulations was proposed to mitigate the time constraints issue of R-CNN: Fast R-CNN [20]. Fast-R$\mathrm{CNN}$ generates feature maps from the input images using CNN instead of feeding the 
region proposals to CNN. The region proposals are then identified and processed into squares. Regions of interest pooling is then applied to reshape the warped regions into a predetermined size, forming the input for an FC. The output layer of the FCs consists of a SoftMax classifier alongside a bounding box regressor. Fast R-CNN only required $0.32 \mathrm{~s}$ for testing and $8.75 \mathrm{~h}$ for training. R-CNN required $47 \mathrm{~s}$ for testing and $84 \mathrm{~h}$ for training. Fast R-CNN achieved an $m A P=39.3 @ 0.5$ on MS COCO dataset with 2000 region proposals and $66 \%$ on PASCAL-VOC-2012 [20]. Fast R-CNN trained VGG16 networks nine $\times$ faster than $\mathrm{R}-\mathrm{CNN}$ and was $213 \times$ faster at test-time.

Fast RCNN and R-CNN both use selective search algorithms to determine the regions of interest (ROI); however, the processing time is a limitation for both methods. Shaoqing Ren et al. [21] proposed an object detection algorithm similar to Fast RCNN, called Faster R-CNN, to overcome this limitation. This algorithm consists of a separate network to predict the region proposals, eliminating the selective search algorithms. Faster R-CNN with a ResNet101 backbone and FPN to extract the feature maps achieved an $m A P=42.7 \%$ with 300 regions when tested on the MS-COCO dataset and 78.8\% mAP on the PASCALVOC-2007 test set.

The previous regions-based detection algorithms perform predictions multiple times for various regions within each single image, which is a time-consuming task; therefore, You Only Look Once (YOLO) has been proposed [24]. YOLO models the detection task as a regression problem instead of using a region proposal. Each image is divided into several grids with two defined bounding boxes, increasing the speed of the detection algorithm. For instance, YOLOv3-320 processed images in real-time at 45 frames per second using Darknet-53 as a backbone, achieving an $m A P=28.2 \%$, while YOLOv3-416 achieved an $m A P=31 \%$, and YOLOv3-608 achieved an $m A P=33 \%$. YOLO can achieve real-time object detection; however, it has several limitations, such as the loss function, which treats the errors induced by small and large bounding boxes equally.

The authors of [27] created the first Fully Convolutional Network (FCN) trained endto-end for image segmentation used in semantic segmentation. Many variants of FCN have been proposed, such as Graph-FCN, which achieved an $m I O U=65.91 \%$ on Pascal-VOC Dataset and an $m I O U=36.64 \%$ with FCN-32s on a PASCAL-Context dataset

The authors of [28] proposed an improvement of the model FCN, called ParseNet. This model improves upon FCN by allowing for global context inclusion in semantic segmentation. Relying on the largest receptive field of the FCN network is not sufficient for providing global context, and the largest empirical receptive field is not sufficient for global capture. ParseNet Baseline and ParseNet trained on the VOC2012 test set achieved a $67.3 \%$ and $69.8 \% \mathrm{mIOU}$, respectively.

The authors of [26] developed DeconvNet, a convolutional neural network (VGG-16) concatenated with a deconvolutional neural network (DNN) for semantic segmentation. The CNN-VGG-16 consists of the pooling needed to generate feature maps from the region in which the proposals are fed, which are then fed to the DNN. The DNN then performs the unpooling to determine the pixel-wise probabilities belonging to each class. The model was evaluated on PASCAL-VOC-2012 and was compared to the state-of-art segmentation algorithms. This model achieved a mean average precision of $69.6 \%$, and some of its variants achieved a mean average precision of around $70 \%$.

The authors of [34] proposed U-Net, a convolutional network for image segmentation, which is built on FCN. U-Net is composed of two paths or two sides: contractive and expansive. The contractive side has an FCN-like architecture extracting feature maps, while the expansive path spatially localizes patterns in the image subject to segmentation. U-Net was the winner of the of the EM segmentation challenge in 2015 and also the ISBI cell tracking challenge of 2015, with an IoU of 0.9203 for the "PhC-U373" dataset and an IoU of 0.7756 for the "DIC-HeLa" dataset.

The authors of [35] proposed DeepLabv3: which improved DeepLab by combining the parallel and cascade modules found within the atrous convolutions. The ResNet architecture was modified to maintain higher resolution feature maps within the same convolution. 
Mask R-CNN can efficiently detect objects while simultaneously generating a highquality segmentation mask for each instance [36]. A CNN network was added to the model parallel to the object detection task to determine the mask or the pixels belonging to the objects. Mask-RCNN does not support real-time analysis and is made up of two blocks. The first block, or backbone, deals with generating region proposals, while the second block, the ROI classifier and Bounding Box Regressor, classifies the regions proposals and generates the bounding boxes and masks.

The backbone consists of a standard convolutional network, typically ResNet50 or RestNet101, which serves as a feature extractor. As the features passing through the backbone network, the images are converted from $1024 \times 1024 \times 3$ (RGB) to a feature map of shape of $32 \times 32 \times 2048$. The new feature map serves as the input for the second block.

Mask R-CNN uses a Feature Pyramid Network (FPN) as an extension that can improve the standard feature extraction. FPN enables access to both lower and higher-level features. The Region Proposal Network (RPN), a type of lightweight neural network, scans over the backbone feature map once it is generated. The regions over which the RPN scans are performed are called anchors; for each anchor, the RPN generates an anchor class consisting of either a foreground class or a background class. The foreground class identifies whether or not there is an object in that box. The background class is the Bounding Box Refinement, which is a foreground anchor. This foreground might not be centered perfectly over the object, and thus, to refine the anchor box, the RPN estimates a change in the box's coordinates, also referred to as delta $(\Delta)$.

The second block of Mask R-CNN runs on the regions of interest proposed by the RPN. The bounding box refinement step in the RPN causes different sizes for the ROI boxes that must be adjusted to the same size; therefore, ROI Align, a new feature of Mask $\mathrm{R}-\mathrm{CNN}$, is used to create a fixed input for the ROI classifier. The stride is not quantized in ROI align, and bi-linear interpolation is considered, while Faster R-CNN uses a quantized stride. The RPN classifier generates two outputs for each ROI: the specific class of the ROI object and the bounding box refinement.

The bounding box refinement works further to refine the location and size of the box to encapsulate the ROI object. The last step of Mask R-CNN is the generation of segmentation masks. The segmentation mask branch consists of a convolutional network, which utilizes the positive regions selected by the ROI classifier and generates a mask. The full architecture along with the output at each step, is illustrated in Figure 1.

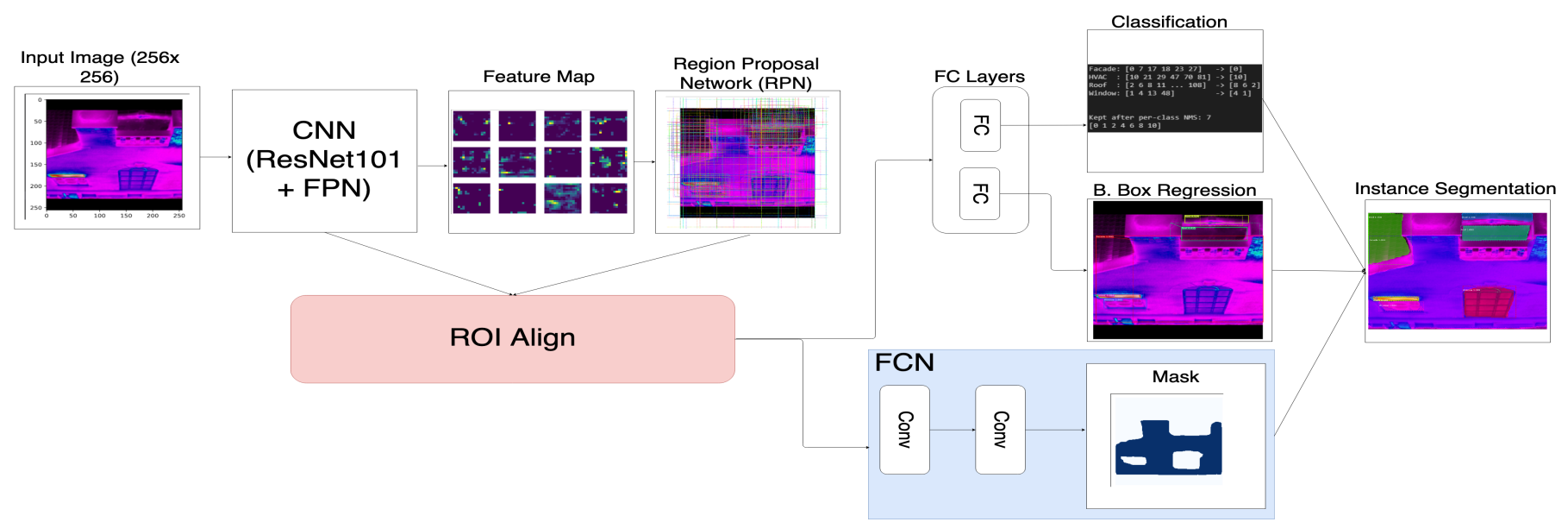

Figure 1. Illustration of the Mask R-CNN architecture with an input image at each stage of detection.

The authors of [30] developed a model, called the Context Encoding Network (EncNet) for instance segmentation. This model is built upon of two building blocks: the first of which consists of a CNN with different backbones, also called ResNet, to generate the feature maps. The output of this last CNN layer is fed into the second block, which is a context encoding module. The Context Encoding Module's outputs are then reshaped 
and processed by a dilated convolution strategy while simultaneously minimizing binary cross-entropy losses and a final pixel-wise loss. The proposed EncNet with Resnet101 achieved an $\mathrm{mIOU}$ of $52.6 \%$ on the PASCAL-Context dataset.

The authors of [33] proposed panoptic segmentation to unify semantic segmentation and instance segmentation. To accomplish panoptic segmentation, the authors proposed a new quality metric to evaluate the overall segmentation. The evaluation metric can then be written as the product of two terms: the segmentation quality (SQ) and one recognition quality (RQ). Machine panoptic segmentation on instance segmentation with Mask RCNN + COCO achieved $P Q^{T h}=54.0,79.4,67.8$ on the Cityscape dataset, and the PSPNet multi-scale achieved a $P Q^{S t}=66.6, S Q^{S t}=82.2$ and $R Q^{S t}=79.3$.

\subsection{Image Clustering: The State of the Art}

Image segmentation techniques have been used to identify, classify, and process regions of interest within colored and red-green-blue (RGB) images and, more recently, within infrared (IR) images. Table 2 provides a brief summary of image clustering methods and their performances.

The authors in [37] used a hybrid approach in their 2016 segmentation paper, that deploys the K-means and the Density Based Spatial Clustering of Application with Noise (DBSCAN) segmentation approaches to identify 'hotspot' regions within IR images of photo voltaic (PV) arrays. Image pixel color values were first normalized and then preprocessed using the K-means method to segment the image into discrete regions of colors. This method creates distinct silhouettes of the various color profiles within the image. DBSCAN was then applied to obtain the pixel regions, which are above a set threshold of saturation in the hue-saturation-value (HSV) color palette.

Table 2. State-of-the-art image clustering techniques.

\begin{tabular}{|c|c|c|}
\hline Clustering Method & Performance & Reference \\
\hline K-means/DBSCAN & $\begin{array}{l}\mathrm{K}=15 \text { was optimal for accuracy and computation time. } \\
\text { Further segmentation by DBSCAN yielded } 136 \text { clusters } \\
\text { for precision. }\end{array}$ & [37] \\
\hline K-means/Threshold & $\begin{array}{c}\mathrm{K}=4 \text { was optimal and yielded a } 99.7 \% \text { accuracy rate in } \\
\text { hotspot detection for an ensemble ML model called } \\
\text { Voting (Naive Bayes + REPTree) }\end{array}$ & [38] \\
\hline Dual Clustering Scheme & $\begin{array}{l}\text { Precision and recall produced averages of } 80 \% \text { and } \\
58.3 \% \text { respectively for } 10 \text { sets of images. }\end{array}$ & [39] \\
\hline IFS/Fuzzy C-means & $\begin{array}{l}\text { Average segmentation and Dice scores of } 99 \% \text { for } \\
\text { varying levels of noise corrupted images. }\end{array}$ & [40] \\
\hline IP-MS & $\begin{array}{l}\text { Average of } 1.4 \mathrm{~s} \text { per sample image in contrast to } 2.3 \mathrm{~s} \\
\text { from the K-means algorithm. Higher accuracy than } \\
\text { K-means in terms of blue color intensity representations. }\end{array}$ & [41] \\
\hline $\begin{array}{l}\text { DEMP-k (Directly Estimated } \\
\text { Misclassification Probabilities) }\end{array}$ & $\begin{array}{l}\text { Tested on digit recognition gives correct classification } \\
\qquad(C P)=0.843 \text {, adjusted Rand Index }(A R)=708 \text { and } \\
\text { computing time }(\tau)=27.03 \mathrm{~s}\end{array}$ & [42] \\
\hline
\end{tabular}

Hajela et al. [38] used a 2D spatio-temporal analysis to detect and cluster regions of crimes, which were identified as hotspots. K-means was the primary approach used to classify different regions within a dataset that contained $(x, y)$ co-ordinates, times, and dates for the events in each image. The number of instances of these K-means clusters were calculated and passed through a threshold to discretely obtain regions of hotspots within an image. This threshold was set based on the number of instances of each cluster and the total number of clusters. When combined with ensemble machine learning models, the use of clustering indicated a marked increase in the accuracy of crime prediction across various crime categories, such as vandalism, bribery, and extortion. 
Another dual clustering method was introduced by Tamilkodi and colleagues [39], where the authors utilized a two-part process to cluster pixels within an image. The RGB query image was pre-processed to gray-scale. A histogram analysis performed based on the intensity or the brightness values of the gray scale image followed. This histogram serves as a one-dimensional space for a K-means based approach to cluster pixels; however, the novelty in this approach is the calculation of two-dimensional gradient with vectors that to point to higher intensity value pixels. This approach also processes these pixels as black or white based on a threshold ' $\mathrm{H}$ '. This method was tested on a set of 1000 images from the Signal and Image Processing Institute (SIPI) and divided into ten groups based on similar content, such as dinosaurs, houses, oceans, horses, and others. Outcomes of $80 \%$ and $58.3 \%$ for the average precision and recall metrics, respectively, were produced by this method.

The authors in [40] utilized a clustering method based on Intuitionistic Fuzzy Set (IFS) theory and Fuzzy C-means (FCM) to segment images generated with magnetic resonance imaging (MRI). The C-means algorithm does not perform well with noise; therefore, the Intuitionistic Fuzzy C-means with Spatial Neighborhood Information (IFCMSNI) method proposed by the authors was used to preserve valuable spatial information through a 'spatial neighborhood information' equation. The outcomes of this method were tested with a gray image MRI dataset with varying levels of noise through metrics, such as the dice score (DS) and the average segmentation accuracy (ASA), which was provided with ground truth data. These metrics indicated a significant improvement over existing methods, such as the Modified Intutionistic Fuzzy C-means (MIFCM) and Fuzzy Local Information C-means (FLICM), in the presence of Rician noise.

The authors in [42] introduced DEMP-k (Directly Estimated Misclassification Probabilities), which is a combination of the HoSC-K-means (Homoscedastic Spherical Components) and hierarchical linkage functions, thereby increasing the speed and performance of the algorithm. Their work proposed a framework for hierarchical merging based on pairwise overlap between components, this was further applied to the K-means algorithm. The model produced the results in Table 2 when tested on a digit recognition dataset.

A novel approach, called the Iterative Partitioning-Mean Shift (IP-MS) was introduced by Naik and colleagues [41], where the number of centroids chosen for each cluster and the number of iterations are key parameters for image segmentation. The image was pre-processed by reducing the noise, transforming the RGB image to a LAB color space, and normalizing the pixel values. The clustering algorithms then classified each pixel by finding the minimum Euclidean distance between pixels for each centroid and calculating the mean distance value for each cluster. Once the mean equals the number of centroids specified by the algorithm, convergence is reached, or the algorithm has successfully executed. The results of this algorithm indicated a marked performance increase in the accuracy and computation time when compared to the K-means algorithm.

\section{Methodology}

In this section, the methodology for data preparation, preprocessing, and evaluation is described. First, we start by describing the data-driven three layered framework to provide the complete picture of the process, then we discuss the building block data preparation. Second, we present K-means and Threshold-Based Clustering for hotspot detection. Last, we describe the U-value analysis using four formulae.

\subsection{Data-Driven Three-Layered Framework}

Infrared thermal imagery is promising due to its extensive features, high performance abilities, and relatively lower cost. The thermal images must be pre-processed and automated before any meaningful information is collected. The image pre-processing includes the removal of unwanted background objects and the detection of inspected elements, such as windows, doors, walls, and other features. The current published research that addresses the terms of automating the methods for background removal, object detection, and 
U-value estimation is limited [43-45]. We, therefore, propose a fully automated three-layer framework for the U-value estimation of a building and its elements. Figure 2 illustrates a data-driven approach for the thermal performance assessment of building envelopes.

The raw thermal imagery captured from various sources, such as aerial or ground measurements, is stored in a data repository or database layer. The images are fed into a pre-processing and automation layer, where a series of background elimination steps are completed, and the critical features from the thermal images are extracted (refer Figure 2). The different building elements, such as doors, roofs, facades, beams, and windows, are annotated and used for training machine learning models on object detection. The heat loss U-values for building envelopes and elements are quantified in the evaluation layer, while influential parameters, such as the emissivity and reflected temperature, are analyzed.

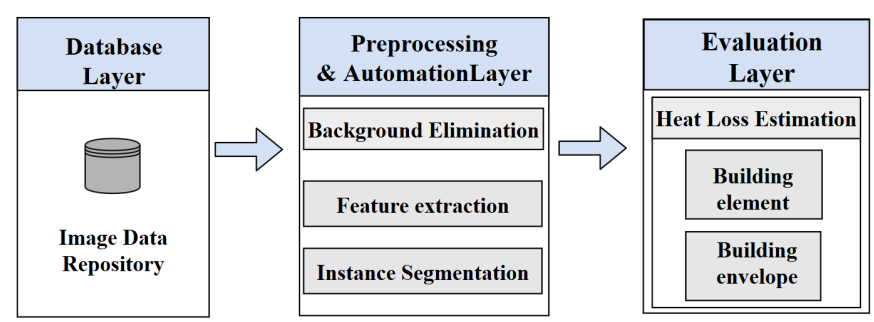

Figure 2. Data-driven approach for thermal performance [4].

\subsection{Dataset Preparation}

The number of objects in the dataset are not distributed equally due to the nature and context of the dataset itself. The frequency of HVACs and doors are far lower when compared to windows and facades for any given building, causing lower detection limit discrepancies for these respective objects. Two tactics were employed to remedy this issue: (1) Each dataset had several augmentation techniques applied to them. These included random color shifts, multiplying the dataset with copies of itself, horizontal and vertical flips, Gaussian blur, and contrast and brightness. The augmentation resulted in the original dataset increasing by a factor of six, on average. (2) Once augmented, datasets were combined based on campus buildings.

This technique drastically increased the objects with low occurrences, and allowed the model to learn and identify these objects more accurately. Each training dataset is listed in Table 3, and a total of four datasets were created for training. Datasets 1 through 3 consisted of images taken at the Museum of Art and Twamley buildings on the UND campus. Dataset 4 consisted of a combination of the Minot State, Wahpeton State, and Bismarck State campuses. The number of augmented instances of facades, windows, roofs, HVACs, and doors are listed in each column of Table 3 . The total number of images used was 42,439.

Table 3. Dataset breakdowns.

\begin{tabular}{ccccccc}
\hline Datasets & $\begin{array}{c}\text { \# of } \\
\text { Facades }\end{array}$ & $\begin{array}{c}\text { \# of } \\
\text { Windows }\end{array}$ & \# of Roofs & $\begin{array}{c}\text { \# of } \\
\text { HVACs }\end{array}$ & $\begin{array}{c}\text { \# of } \\
\text { Doors }\end{array}$ & $\begin{array}{c}\text { Total } \\
\text { Images }\end{array}$ \\
\hline Dataset 1 & 2060 & 1109 & 634 & 343 & 100 & 2562 \\
Dataset 2 & 10,190 & 13,987 & 1894 & 0 & 126 & 10,971 \\
Dataset 3 & 2576 & 5207 & 492 & 2085 & 282 & 2541 \\
Dataset 4 & 26,217 & 18,684 & 11,747 & 1616 & 6448 & 26152 \\
Test Dataset & 207 & 176 & 95 & 38 & 43 & 213 \\
Total & 41,250 & 39,163 & 14,862 & 4082 & 6999 & 42,439 \\
\hline
\end{tabular}

\subsection{Thermal Hotspot Detection via Clustering Techniques}

\subsubsection{Threshold-Based Clustering (TBC)}

Once the window- or facade-only pixels were obtained for a particular image, they were classified as areas of interest and passed through a threshold to obtain hotspot relevance. The initial testing of this approach applied the use of a static threshold based 
on percentiles; however, this approach was discontinued due to its inability to adapt and identify hotspots under extreme variations in the input surface temperatures. Figure 3 illustrates a flowchart for the two clustering algorithms.

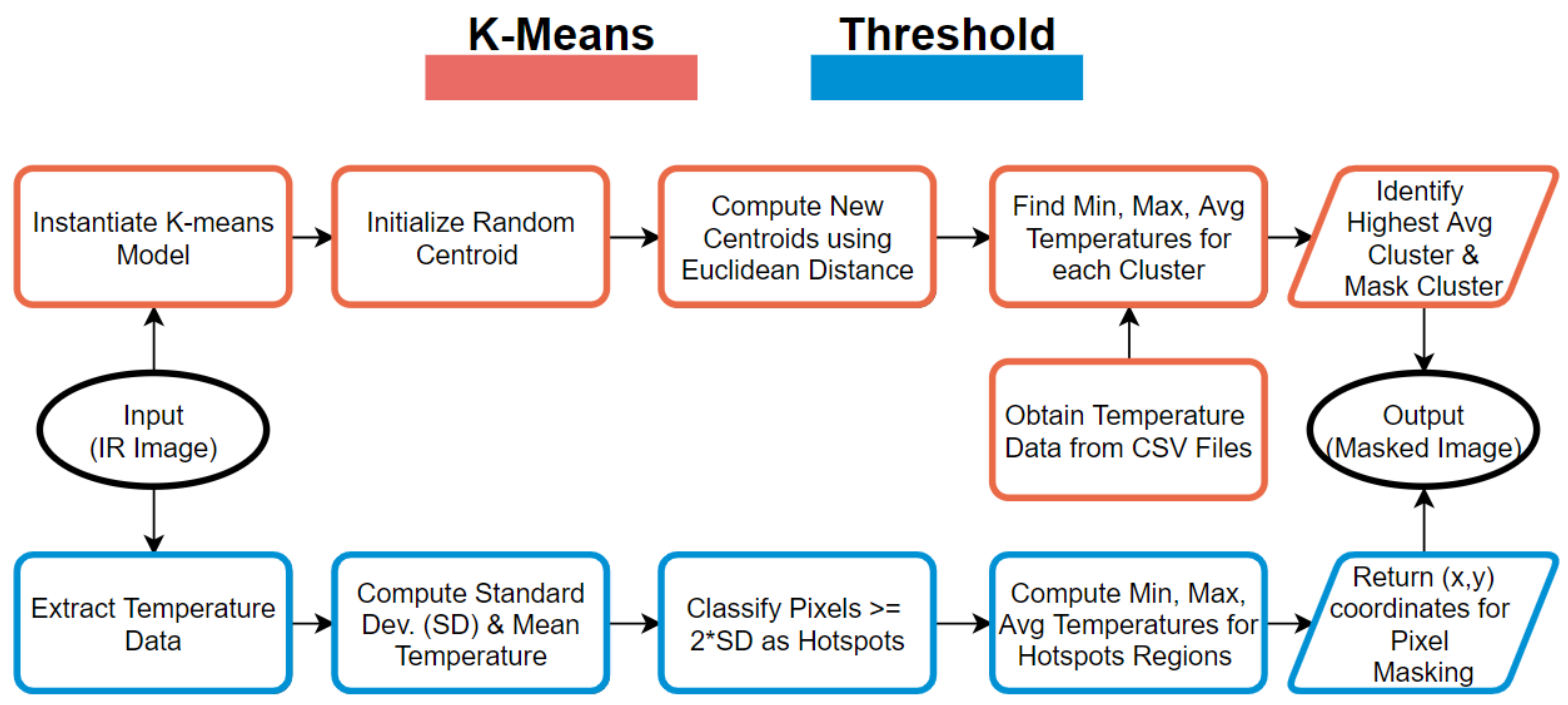

Figure 3. Flowchart of thermal image clustering using K-means and Threshold-Based Clustering.

Threshold-Based Clustering is based on the objects' mean temperature $(\mu)$ and standard deviation $(\sigma)$, considering object temperatures of $>=2 \sigma$ and processing the pixels corresponding to those temperatures. The following piece-wise functions can be represented, mathematically, as

$$
\begin{gathered}
I_{x, y}= \begin{cases}1, & \text { if } t_{x, y}>=2 \sigma+\mu\left[T_{o}\right] \\
0, & \text { if } t_{x, y}<2 \sigma+\mu\left[T_{o}\right]\end{cases} \\
(\hat{R}, \hat{G}, \hat{B})_{x, y}^{\text {pixel }}= \begin{cases}(255,0,0)_{x, y}^{\text {pixel }}, & \text { if } I_{x, y}=1 \\
(R, G, B)_{x, y}^{\text {pixel }}, & \text { if } I_{x, y}=0\end{cases}
\end{gathered}
$$

where $I_{x, y}$ is the Identity Matrix that holds hotspot (binary 1) and non-hotspot (binary 0) pixels, $t_{x, y}$ is the pixel at co-ordinates $(x, y)$, and $T_{o}$ is the set of all pixels within an object of interest, such as the walls or windows. $(\hat{R}, \hat{G}, \hat{B})_{x, y}^{\text {pixel }}$ represents the RGB pixels at co-ordinates $(x, y)$ that are colored red for a detected hotspot and unchanged if not.

The thresholds on surface temperature were evaluated using Infrared Camera Inc. (ICI) thermal imaging software. These thresholds are visually intuitive: when looking at the raw thermal image, the regions of longer wavelengths in the visible light spectrum, represented in red, are the areas with a greater density of pixels denoting a higher temperature. The user can identify these regions as hotspots and use a shaping tool to draw boundaries for segregating the image into hotspot sections. This activity is a tedious and inaccurate process that yields only the maximum, minimum, and average temperatures. These regions are identified on a granular level using TBC, where each pixel is analyzed for its suitability as a hotspot. Hotspot regions are higher temperatures, and due to heat dissipation, are considered regions of significance when estimating U-values.

\subsubsection{Hotspot Detection Using K-Means}

$\mathrm{K}$-means is a common data mining approach to group ' $\mathrm{N}$ ' observations into ' $\mathrm{K}$ ' clusters with the nearest mean, or centroid of a cluster, by minimizing the squared Euclidean distances. We evaluated groupings of surface temperature observations from the captured 
thermal images, which were each divided into ' $K$ ' clusters based on different colors formed by the combinations of color channels. The surface temperature observations were compared with TBC for the reliability of the clustering method. This method was created using the Scikit-learn Library [46].

K-means segments an image into different clusters based on colors. This approach is based on the idea that the colors in a thermal image represent different temperatures regions. This method can be further divided into two parts: segmentation and hotspot identification.

We calculated the minimum, maximum, and average temperatures for each cluster using the pixel temperature data from the CSVs when using hotspot identification. The methodology of the clustering phases is further explained in Algorithm 1.

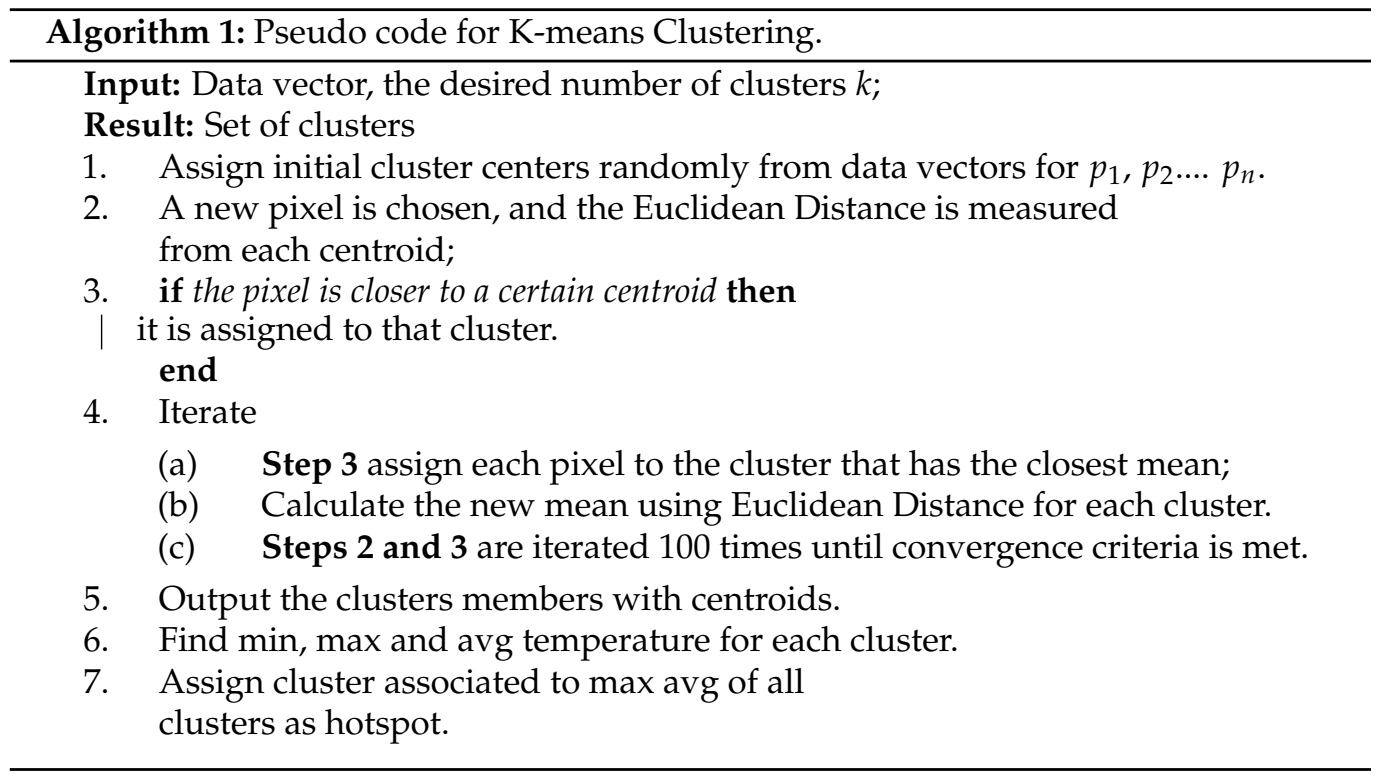

An image is a $3 D$ vector of colors comprised of combinations of (i.e., Red, Green, and Blue) with value ranges from $0 \longrightarrow 255$ for each channel. A cluster is determined by grouping those pixels with the least Euclidean Distance from the chosen centroid. Considering two pixels $i$ and $j$ with values $\left(R_{i}, G_{i}, B_{i}\right)$ and $\left(R_{j}, G_{j}, B_{j}\right)$. The Euclidean distance $D_{i j}$ between them can be further computed as follows:

$$
D_{i j}=\sqrt{\left(R_{i}-R_{j}\right)^{2}+\left(G_{i}-G_{j}\right)^{2}+\left(B_{i}-B_{j}\right)^{2}}
$$

K-means proceeds by selecting random pixels as centroids. This method of initializing the centroid has been reformed over the years with a better seeding method called Kmeans $^{++}$as stated by Arthur et al [47]. The main goal for K-means is to reduce the Sum of Squared Distance for each cluster at every iteration. This is achieved by selecting new pixels as centroids and calculating the Euclidean distance with their respective cluster members. Equation (4) shows that, for each cluster from $n=1$ to $N$, a cluster is chosen, and the Euclidean Distance between a pixel in $n$ and the centroid is calculated. This is iterated over and over for every cluster $n$ to minimize $J$ by selecting new pixels $P_{n}$ and centroids $C_{n}$.

$$
J_{\min }=\sum_{n=1}^{N} \sum_{P \in n} D_{C_{n} P_{n}}
$$

Computing the $\mathrm{K}$-means for such large datasets raises the question of selecting the range of clusters. This problem is solved using the Elbow Method [48], which considers the sum of distances between cluster centers (and their respective cluster members) versus 
the number of clusters. For each dataset, this had to be computed to obtain a range of $k$ or an optimal number of clusters that are ideal for that data set.

\subsection{U-Value Estimation}

Various U-value measurement-based methods have been used in the literature to estimate thermal performance. According to the Stefan-Boltzmann law, the radiative heat varies with the fourth power of temperature difference [49].

The U-value estimation for the external surface was carried out while considering the wind velocity using the following equation in [50-52]

$$
U_{1}=\frac{\epsilon \sigma\left(T_{s e}^{4}-T_{a e}^{4}\right)+3.805 v\left(T_{s e}-T_{a e}\right)}{T_{a i}-T_{a e}}
$$

where $v$ is the velocity of the external wind, $T_{s e}$ denotes the external surface temperature, $T_{a i}$ denotes the internal air temperature, and $T_{a e}$ denotes the external air temperature. The radiative heat component is similar to the Stefan-Boltzmann equation [49] and the convective term is derived from Jargon's formula [53].

Fokaides et al. [54] estimated the U-value according to the Stefan-Boltzmann law as follows.

$$
U_{2}=\frac{4 \epsilon \sigma T_{s}^{3}\left(T_{s i}-T_{r e f l}\right)+\alpha_{c}\left(T_{s i}-T_{a i}\right)}{T_{a i}-T_{a e}}
$$

where the wall emissivity and convective heat coefficient is denoted by $\varepsilon$ and $\alpha_{c}$, respectively. In addition, $\sigma$ denotes the Stefan-Boltzmann constant and $T_{i n}, T_{o u t}, T_{s i}$, and $T_{r e f l}$ denote the internal, external, internal surface, and reflexive wall temperatures respectively. The surface temperature is denoted by $T_{s}$. The mean temperature was introduced in Equation (7) by Madding et al. [55] to replace the surface temperature in Equation (6) to estimate the U-values as follows:

$$
U_{3}=\frac{4 \epsilon \sigma T_{m}^{3}\left(T_{s i}-T_{r e f l}\right)+\alpha_{c}\left(T_{s i}-T_{a i}\right)}{T_{a i}-T_{a e}}
$$

where $T_{m}=\frac{T_{s}+T_{\text {refl }}}{2}$ denotes the mean temperature, and $\epsilon$ and $\sigma$ denote the emissivity and Stefan-Boltzmann constant, respectively. In this equation, the reflective temperature is subtracted from the surface temperature. Spectrum emissivities of 0.75 for the wall and 1.0 for windows were taken. The material for walls was considered to be fire brick, and the windows were classified to be of a smooth glass material, which had emissivities in the range of 0.92-0.96 [55-57]; however, for the purposes of this paper, glass was considered to be a perfect black body (in the context of U-value estimation) as there would be negligible differences if we use Equation (5) to calculate the U-values. The convective coefficient $\alpha_{c}$ depends on various factors, including the height of the wall and the temperature difference shown in Equation (8).

$$
\alpha_{c}=c_{1}{\frac{\left(T_{s i}-T_{a i}\right)^{\frac{1}{4}}}{L}}^{-}
$$

where $L$ is the height of the wall and the value of $c_{1}$ varies from 1.31 to 1.42. Equation (8) is obtained from reference [55], where $c_{1}$ varied from 0.25 to 1.42 depending on the type of air-flow. When the wall is tall, and the temperature difference is large, the coefficient can change depending on the type of flow (turbulent or laminar). Figure 4 illustrates the deviation of the U-value with respect to acceptable $c_{1}$ values within the range of 0.25 to 1.42. The acceptable range of $c_{1}$ values was deduced empirically. Several types of objects and their corresponding $\mathrm{U}$-values were plotted with $c_{1}$ values varying from 0.25 to 1.42 . These $\mathrm{U}$-values were then converted to $\mathrm{BTU} / \mathrm{hr} \mathrm{ft}^{2 \circ} \mathrm{F}$ first and then to $\mathrm{W} / \mathrm{m}^{2} \cdot \mathrm{K}$ before 
being compared to ASHRAE standards, which allowed us to identify which constants aligned with the expected values.

We consider $U_{c}$ as the average of the $U$-values, calculated previously as

$$
U_{c}=\frac{U_{1}+U_{2}+U_{3}}{3}
$$

The IR Flash Pro software was used to extract the temperature information into a CSV file containing $512 \times 640$ cells. The building height information was obtained from the architectural diagram.

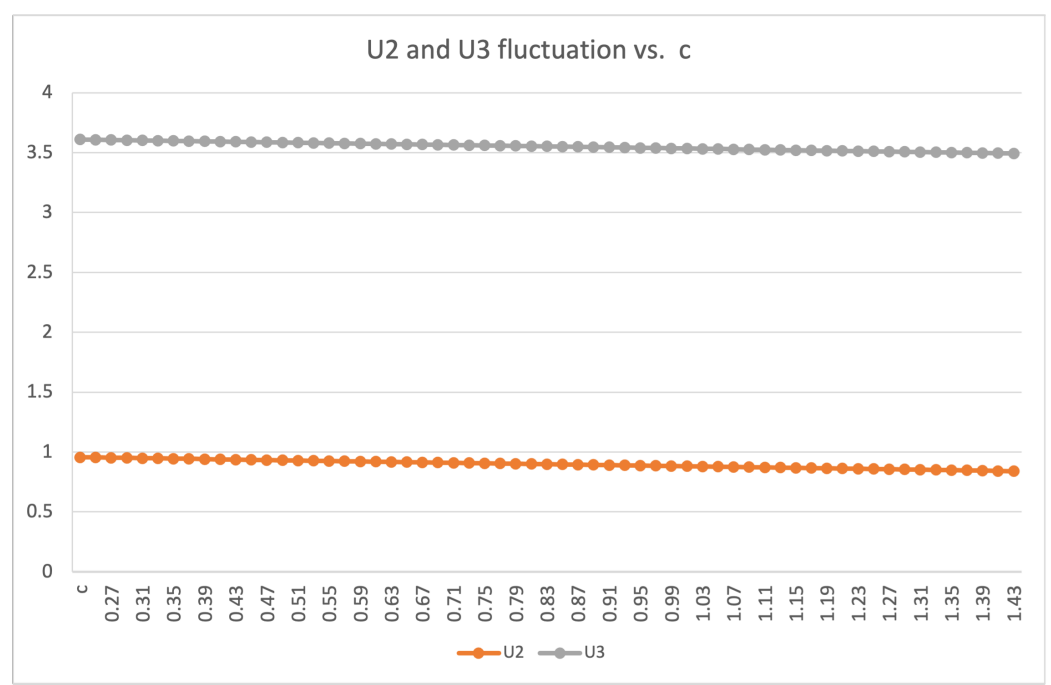

Figure 4. Calculated U-values based on variation of $c_{1}$.

\section{Results and Discussion}

In this section, examples of results are presented and discussed. We start by presenting an evaluation of computer vision algorithms for detection and instance segmentation. Then, we present some examples of results related to clustering and their analysis as well as their discussion. In the last part, we present the U-value estimation as well as examples of the obtained results using different formulas.

\subsection{Evaluation Metrics}

In order to evaluate the performance of deep-learning-based thermal image instance segmentation, a confusion matrix can be used, and, from this, several other metrics can be derived. Table 4 shows the confusion matrix and is defined to show the model's ability to correctly and incorrectly identify objects.

Table 4. Confusion matrix.

\begin{tabular}{ccc}
\hline & Positive (1) & Negative (0) \\
\hline Positive (1) & TP & TN \\
Negative (0) & FP & FN \\
\hline
\end{tabular}

One of the popular metrics used for measuring the accuracy of object detection is the average precision (AP). The average precision computes the AP value for a recall value of 0 to 1 . The precision quantifies the percentage of correct predictions. Recall measures how well the positive values are detected. The mathematical definitions of precision and recall are as follows:

$$
\text { Precision }=\frac{T P}{(T P+F P)}
$$




$$
\text { Recall }=\frac{T P}{(T P+F N)}
$$

where $T P$ is true positive, $F P$ is false positive, and $F N$ is false negative.

In order to determine true positives, the intersection over union is used (Figure 5). The IoU measures the area of overlap between the ground truth and prediction boundaries. Mathematically, the intersection over union is calculated as the ratio of the area of the overlap to the area of union.

$$
\mathrm{IoU}=\frac{A_{0}}{A_{u}}
$$

where $A_{o}$ and $A_{u}$ are the areas of overlap and union respectively. If the $I o U$ is greater than the threshold, the detection is considered correct, otherwise, it is a false detection.

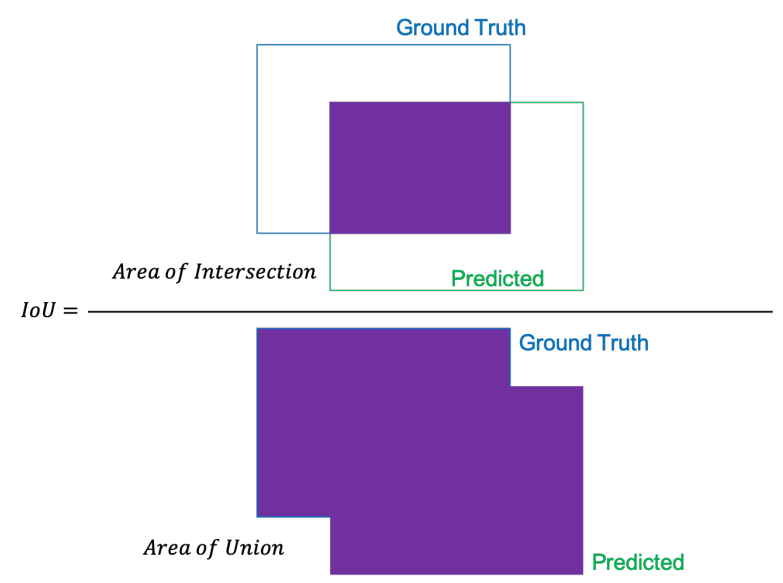

Figure 5. Computing the Intersection of Union, IoU, calculated by dividing the area of overlap between the bounding boxes by the area of union.

The general definition of the average precision is finding the area under the precisionrecall curve.

$$
\int_{0}^{1} p(t) d t
$$

The interpolated AP is calculated by replacing $p(t)$ in Equation (13) by

$$
\int_{0}^{1} \max _{r \geq t}(r) d t
$$

\subsection{Results of Detection and Instance Segmentation Based on Deep Learning}

The models were trained on a machine containing an Intel Core i9-9920X with four Nvidia GeForce RTX 2080 Ti GPU's. Each card consists of 11 GB GDDR6 memory and 544 tensor cores. Each model was trained on one GPU with different configurations, and the model with the best metrics was chosen to be trained on by the next dataset. Adjusted configurations include the batch size, learning rate, and epochs. Table 5 illustrates the learning rate, number of epochs, and training time for each dataset. For the first three datasets, we noticed that reducing the learning rate by a factor of ten at each subsequent training session helped to improve the model accuracy.

This improved model accuracy was due to the first three datasets containing data from UND campus buildings, which have similar architectures. Dataset four consisted of several different campuses, and thus a higher learning rate yielded better results. The training time for each dataset was proportional to the number of images found within them. Dataset four consisted of three different campuses, since training on each individual campus degraded the model performance. 
Table 5. Hyper parameters of the best performing model at each training session (Mask R-CNN).

\begin{tabular}{cccc}
\hline Training Dataset & Learning Rate & Epochs & Training Time \\
\hline DS1 & 0.001 & 75 & $7 \mathrm{~h} 35 \mathrm{~m}$ \\
DS2 & 0.0001 & 100 & $30 \mathrm{~h} 38 \mathrm{~m}$ \\
DS3 & 0.00001 & 150 & $21 \mathrm{~h} 47 \mathrm{~m}$ \\
DS4 & 0.0001 & 200 & $288 \mathrm{~h} 35 \mathrm{~m}$ \\
\hline
\end{tabular}

The test dataset was generated with 10 images from each campus. These 70 images were subsequently deleted from their original datasets to eliminate them from the machine learning process. Similar augmentation techniques were applied to the test dataset to increase the size and test the models fitness. After the augmentation process, the size of the dataset increased to 213 images. The breakdown of dataset is provided in Table 3, which breaks down each dataset by the number of instances in each class within it along with the cumulative values.

Table 6 shows the average precision and the mIoU of the three object detection models and one instance segmentation model. The models were trained on and validated using thermal images captured by the ICI Mirage 640 camera and the ratio to training verses testing was 90:10. A total of five classes were identified for the models to train on: Windows, Facades, Roofs, HVACs, and Doors. The models were evaluated after each training session; however, the results presented are after the final training session. The Average Precision at thresholds of $25 \%, 50 \%$, and $75 \%$ were recorded, and the results show that Mask R-CNN outperformed the other three models for all thresholds. The other three models especially suffered at the $75 \%$ threshold, which indicates that the models are only able to identify a few objects with high confidence.

The feature maps generated were not adequately able to capture the patterns in this thermal dataset leading to low confidence in the models. The three object detection models suffered in estimating the size of objects as well. This is shown in the low mIoU scores achieved by the models. It is also beneficial to compare the pure object detection models against themselves. All three object detection models utilized Faster R-CNN with different backbone architectures.

These models were also evaluated to a similar AP score at all thresholds; however, the Inception ResNetV2 backbone performed slightly better. This is prevalent in the slightly higher AP at 0.75 . The mIoU of both the Inception ResNetV2 and ResNet 50 were the same at 0.34; however, the Inception ResNetV2 backbone achieved higher results for windows, roofs, doors, and HVAC systems while the ResNet 50 model achieved a higher facade evaluation. Overall, Mask R-CNN achieved an average mIoU of 0.66 with Facade and Roofs having the highest overlap of 0.73 and 0.67 , respectively.

The Mask R-CNN model was selected for two main reasons. When quantifying heat loss on buildings, the U-value equations are extremely sensitive to small shifts in temperature and emissivity. This sensitivity required our detection to be precise, with traditional bounding box detection being insufficient for our purposes. Using bounding boxes allows for noise to be introduced since the object contour is not calculated. Instance Segmentation allows us to classify accurate results in greater detail to match ASHRAE standards. Emissivity plays a large role within each of the U-value equations and changes based on the material composition of the object in question. Based on the classification and composition, the emissivity value was looked up on multiple infrared emissivity tables.

The Mask R-CNN model also yielded better results (please see Figure 6) when compared to the Faster R-CNN models with different backbones. Both object detection and instance segmentation models were trained in a similar fashion with varying learning rate decay for the first three datasets, and higher decay for the fourth dataset. The number of epochs was held constant for all models. With the introduction of the mask branch, the Mask R-CNN model took longer to train with more favorable results. We, therefore, selected the Mask R-CNN model. 
Table 6. The average precision of computer vision algorithms trained and tested on OGI thermal images with three different threshold values for five classes: windows, facades, roofs, HVACs, and doors.

\begin{tabular}{|c|c|c|c|c|c|c|}
\hline Classes & $A P^{0.25}$ & $A P^{0.5}$ & $A P^{0.75}$ & Intersection & Union & $m I o U$ \\
\hline Window & 0.39 & 0.39 & 0.18 & $12,648.28$ & $27,679.36$ & 0.45 \\
\hline Facades & 0.34 & 0.31 & 0.12 & $39,487.61$ & $118,876.24$ & 0.33 \\
\hline Roof & 0.41 & 0.31 & 0.07 & $20,042.73$ & $55,555.64$ & 0.36 \\
\hline HVAC & 0.27 & 0.27 & 0.09 & 192.43 & 1085.39 & 0.17 \\
\hline Door & 0.06 & 0.06 & 0 & 414.12 & 6955.88 & 0.05 \\
\hline $\begin{array}{c}\text { Faster R-CNN Inception } \\
\text { ResNetV2 } \\
\end{array}$ & 0.29 & 0.27 & 0.09 & $15,503,247$ & $44,762,491$ & 0.34 \\
\hline Window & 0.26 & 0.10 & 0.03 & $10,011.70$ & $28,303.41$ & 0.35 \\
\hline Facades & 0.41 & 0.32 & 0.05 & $29,035.47$ & $126,352.91$ & 0.22 \\
\hline Roof & 0.38 & 0.26 & 0.13 & $16,002.26$ & $59,677.54$ & 0.26 \\
\hline HVAC & 0.09 & 0 & 0 & 314.15 & 1927.03 & 0.16 \\
\hline Door & 0.35 & 0.26 & 0 & 674.93 & 6983.51 & 0.09 \\
\hline Faster R-CNN Inception V2 & 0.30 & 0.19 & 0.04 & $11,936,209$ & $47,551,061$ & 0.25 \\
\hline Window & 0.28 & 0.19 & 0.12 & 10326.40 & $29,569.77$ & 0.34 \\
\hline Facades & 0.31 & 0.26 & 0.07 & $49,439.15$ & $129,971.74$ & 0.38 \\
\hline Roof & 0.42 & 0.16 & 0.012 & $16,804.96$ & $53,316.74$ & 0.31 \\
\hline HVAC & 0.23 & 0 & 0.012 & 117.57 & 1011.42 & 0.11 \\
\hline Door & 0 & 0 & 0 & 411.18 & 8357.96 & 0.04 \\
\hline Faster R-CNN ResNet 50 & 0.25 & 0.20 & 0.07 & $16,422,148$ & $47,334,488$ & 0.34 \\
\hline Window & 0.70 & 0.69 & 0.44 & $21,545.78$ & $39,170.45$ & 0.55 \\
\hline Facade & 0.81 & 0.79 & 0.67 & $131,982.81$ & $179,617.92$ & 0.73 \\
\hline Roof & 0.67 & 0.67 & 0.67 & $53,879.35$ & $80,260.40$ & 0.67 \\
\hline HVAC & 0.27 & 0.27 & 0.18 & 508.23 & 4501.80 & 0.11 \\
\hline Door & 0.67 & 0.67 & 0.68 & 2815.73 & $11,665.11$ & 0.24 \\
\hline Mask R-CNN & 0.62 & 0.62 & 0.53 & $14,961,967$ & $22,380,315$ & 0.66 \\
\hline
\end{tabular}

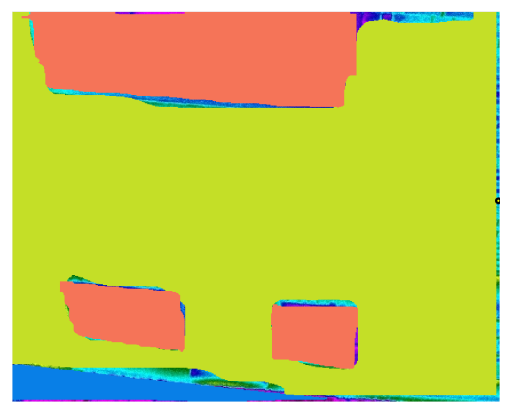

(a) Example of model inference on the UND Museum Building. The metrics achieved were $0.67 @ \mathrm{mAP} 0.25$, mAP 0.5 , and mAP 0.75 with a mIoU of .82

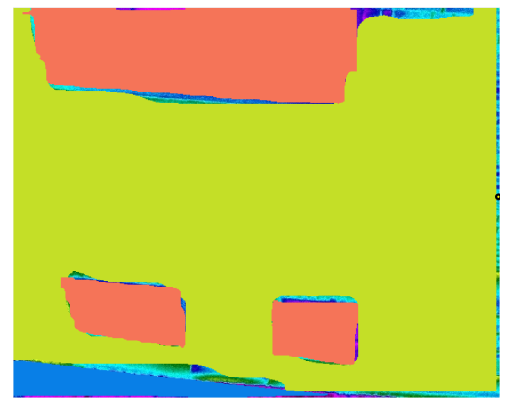

(b) Example of model inference on the Wahpeton State Building. The metrics achieved were $0.76 @$ mAP 0.25, $0.64 @$ mAP 0.5, and $0.60 @$ mAP 0.75 with a mIoU of 0.65

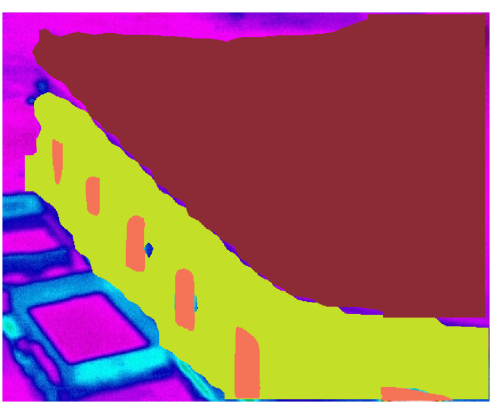

(c) Example of model inference on the Minot State Building. The metrics achieved were $0.91 @$ mAP 0.25, mAP 0.5 , and mAP 0.75 with a mIoU of 0.92

Figure 6. Examples of images of building segmented using Mask-RCNN trained on the heat loss dataset. 


\subsection{Clustering Performance}

Metrics, such as the Silhouette Coefficient and Davis-Bouldin Index, were evaluated for K-means. As explained by [58], the Silhouette Coefficient is a popular metric to find the quality of clustering. It is a measure of how a particular data point or pixel value in our use case is similar to its own cluster compared to other clusters. The coefficient ranges from -1 to 1 , where a positive value signifies that the clustering was well performed. Davis et al. [59] introduced the Davis-Bouldin Index. This metric is an average of the similarity for a cluster to its nearest cluster, which is a ratio of the intra-cluster distance to the inter-cluster distance. The minimum score is 0 , with lower values indicating better clustering. The Silhouette Coefficient for the Museum of Art and Twamley buildings were 0.71 and 0.68, respectively. The Davis-Bouldin Index for the Museum of Art and Twamley buildings dataset were 0.81 and 0.75 , respectively.

Figure 7 highlights the hotspot regions in discrete red and yellow sub-regions for a window (Window 1) at the UND Museum of Art and Twamley buildings using the TBC and K-means approaches, respectively.

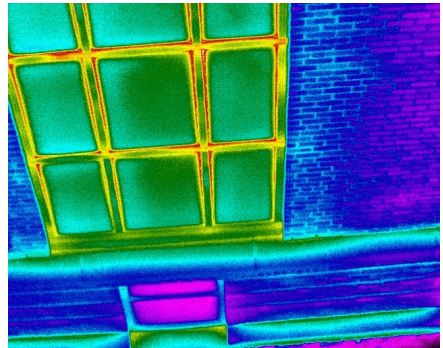

(a) Museum Morning (Raw)

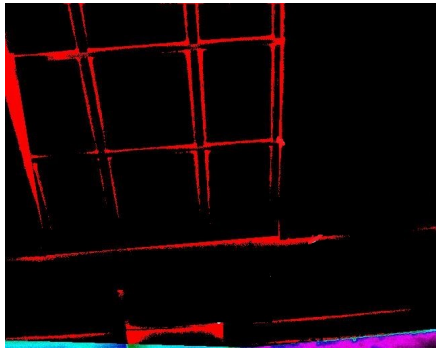

(e) Museum Morning (Threshold)

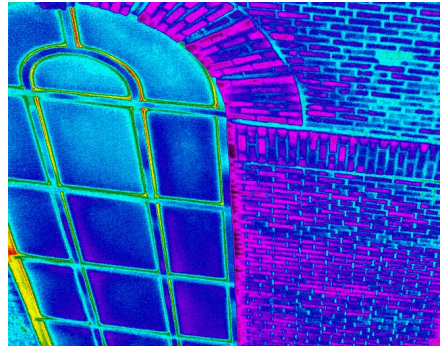

(b) Museum Evening (Raw)

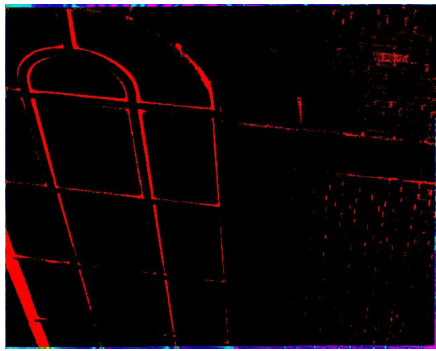

(f) Museum Evening (Threshold)

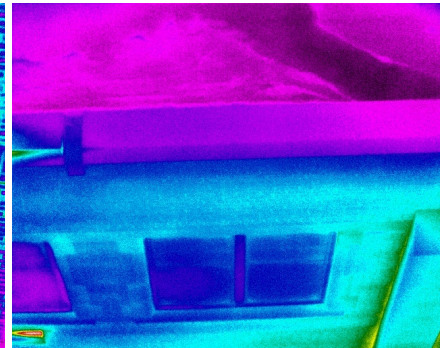

(c) Twamley Morning (Raw)

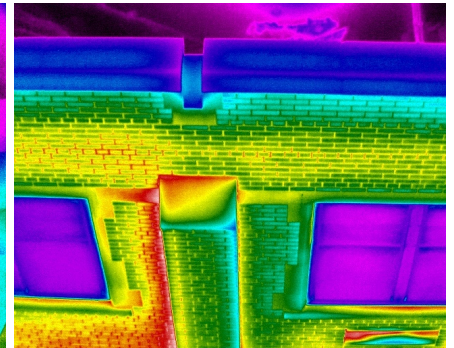

(d) Twamley Evening (Raw)
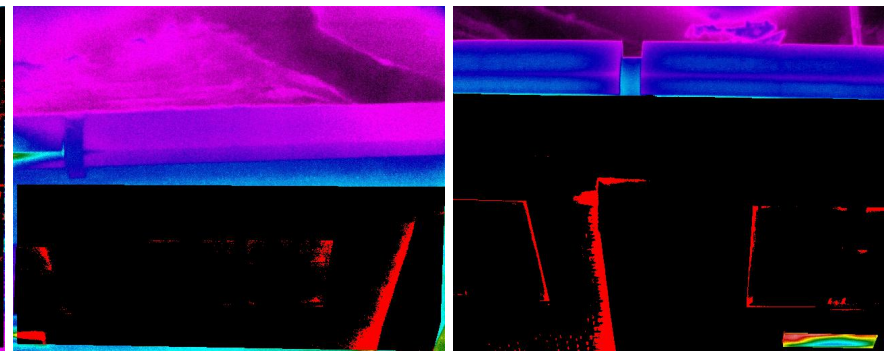

(g) Twamley Morning (Threshold) (h) Twamley Evening (Threshold)

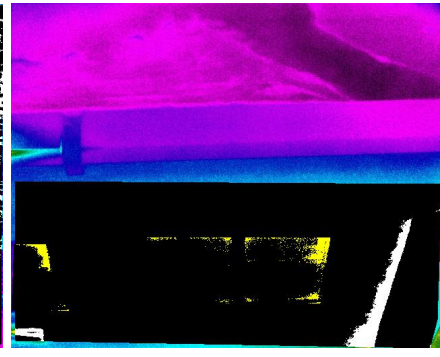

(k) Twamley Morning (K-means)

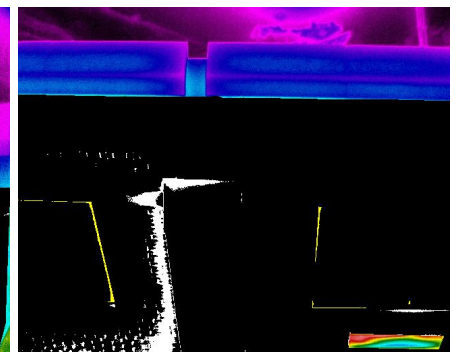

(1) Twamley Evening (K-means)

(i) Museum Morning (K-means)

(j) Museum Evening (K-means)

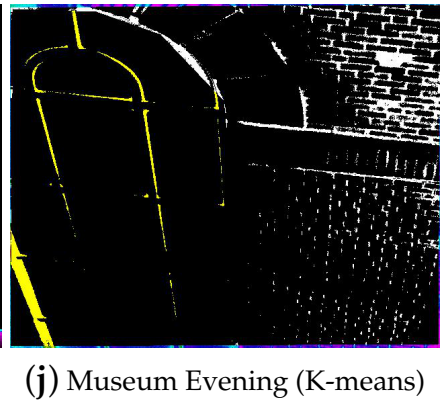

Figure 7. Raw (a-d) and hotspot (e-1) images for two particular windows of Museum and Twamley over the morning, afternoon, and evening.

Table 7 compares the two clustering methods and establishes a comparison metric (called the overlap) for windows and facades, respectively. The overlap metric is the ratio of overlapped hotspot pixels or similar pixels identified individually by the Threshold and Kmeans approaches to the total number of hotspot pixels identified by each of the clustering approaches. Keeping a maximum error of $10 \%$, there were five instances when the two 
clustering methods can be considered to be in agreement. However, this is marginally short of a $50 \%$ split and cannot be used to definitively conclude a consensus. Two other metrics were considered for comparison and are discussed in the following two paragraphs.

Table 7. Hotspot pixel overlapping (\%) for windows and walls.

\begin{tabular}{ccccccccc}
\hline Object of Interest & \multicolumn{4}{c}{ Windows } & \multicolumn{3}{c}{ Walls } \\
\hline Buildings & \multicolumn{2}{c}{ Museum } & \multicolumn{2}{c}{ Twamley } & \multicolumn{2}{c}{ Museum } & \multicolumn{2}{c}{ Twamley } \\
\hline Duration & Threshold & K-Means & Threshold & K-Means & Threshold & K-Means & Threshold & K-Means \\
\hline Morning & $88.2 \%$ & $64.2 \%$ & $86 \%$ & $93 \%$ & $69.8 \%$ & $76.8 \%$ & $79 \%$ & $70 \%$ \\
Evening & $82.8 \%$ & $71.8 \%$ & $43 \%$ & $93.4 \%$ & $71.4 \%$ & $42.1 \%$ & $72 \%$ & $40.4 \%$ \\
Afternoon & $77.9 \%$ & $73.1 \%$ & $82 \%$ & $43 \%$ & $72.8 \%$ & $64.2 \%$ & $34 \%$ & $35 \%$ \\
\hline
\end{tabular}

Figure $8 \mathrm{~b}$ depicts the minimum, maximum, and average surface temperatures for six clusters created in the segmentation phase. The cluster with the highest average temperature (such as cluster 3 Figure $8 b$ ) was chosen as the hotspot. Figure $8 \mathbf{b}$ shows the Elbow evaluation for an image from the museum dataset. From the graph in Figure 8 , we can identify the $k$ value for the walls to be somewhere in the range between 3 to 6 . After the seventh cluster, it was evident that there were no such changes in the squared distance. The $\mathrm{K}$ value of six was chosen using the temperature $\mathrm{TBC}$ as the ground truth because of the hotspot evaluation technique involving pixel temperatures. Computing the K-means to six clusters yielded results with few deviations with respect to Average Hotspot Temperature and Density of Hotspot from TBC. A value of $K=5$ yielded results similar to the TBC for the windows. The performances of the clustering techniques across different parameters are listed in Table 8.

Table 8 compares the results obtained by the clustering approaches based on a fixed set of five parameters across the morning, afternoon, and evening time periods. The "Density (Hotspot)" measure is a ratio of the number of hotspot pixels to the total number of pixels within the entire surface being measured, such as windows or facades. Similarly, the "Average Temperature (Hotspot)" measure is the average temperature of the hotspot regions identified by each of the clustering methods. The largest discrepancy can be seen when comparing the density metric between the two clustering approaches during the afternoon for Twamley. This discrepancy was caused by the incidence of solar radiation on Twamley's surface.

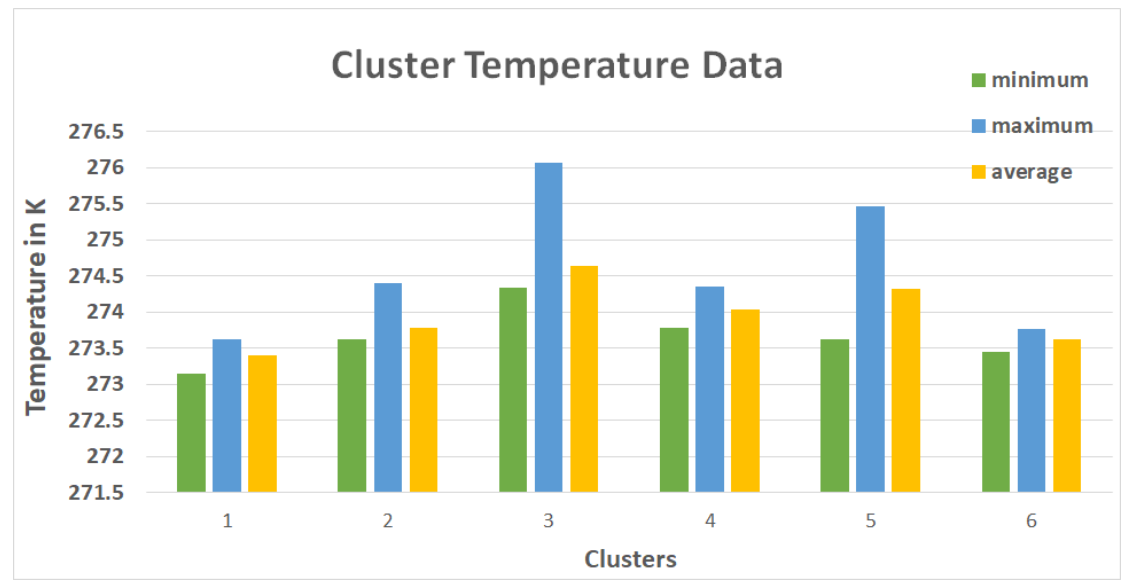

(a) Min, max, and average temperatures for different clusters.

Figure 8. Cont. 


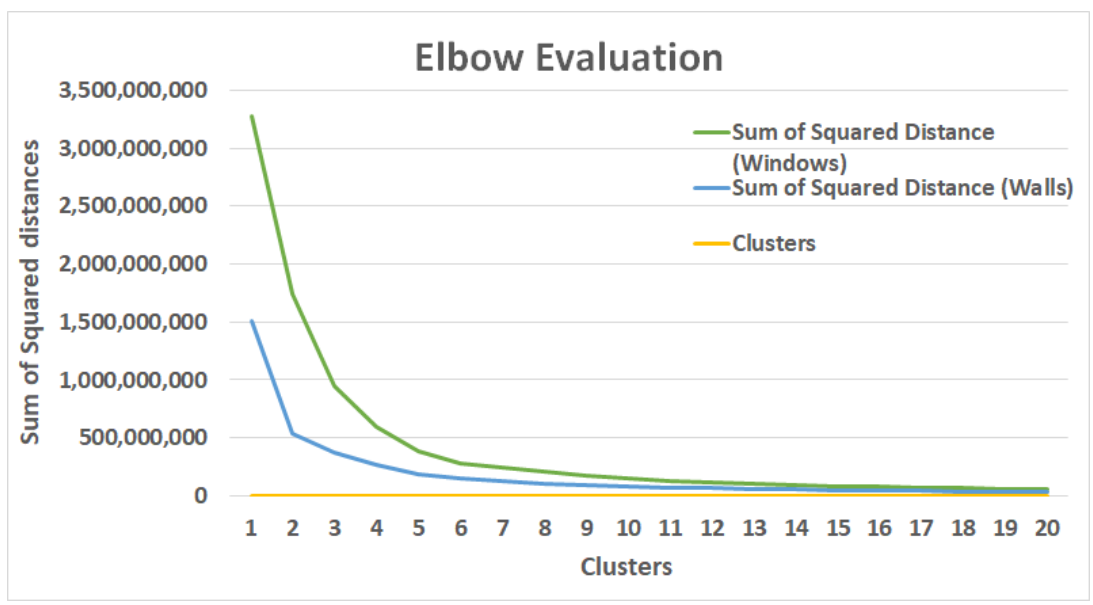

(b) Elbow method evaluation for the images in Dataset 1.

Figure 8. The K-means-optimal cluster evaluation and temperature results.

For a fair comparison, the average hotspot temperatures across different time frames can be taken into account. The values obtained for these measures are consistent across the morning (both the buildings), afternoon (museum only), and evening (both the buildings) time periods with average hotspot temperature differences between 0.01 and 0.48 degrees Kelvin and can be considered negligible. The afternoon duration for Twamley is not considered because, as mentioned earlier, a skewing factor was introduced by solar irradiance. It should also be noted that the temperature values obtained by the sixth cluster from the K-means approach were the most accurate values for the Museum dataset. Accuracy here was assessed when the values of the K-means approach were closest to the values from the TBC as temperature values in the latter were extracted directly from each pixel and, thus, are taken to be the ground truth.

In order to obtain the U-value for UND's buildings, the Stephen-Boltzmann constant $\sigma$ was replaced by $5.67 \times 10^{-8} \mathrm{Wm}^{-2} \mathrm{~K}^{-4}$ in Equation (5) in addition to the spectrum emissivities mentioned earlier. Tables 9 and 10 show the U-values and related parameters for UND's Museum and Twamley buildings). Each of these tables contains the investigated building elements, number of images considered, min-max-average surface temperature captured from the thermal images, air temperature obtained from weather data, $U_{1}, U_{2}$, $U_{3}$, and $U_{c}$ (first obtained in BTU $/ \mathrm{ft}^{2} \mathrm{~h}^{\circ} \mathrm{F}$ and then converted to $\mathrm{W} / \mathrm{m}^{2} \cdot \mathrm{K}$ and where $\left.U_{c}=\frac{U_{1}+U_{2}+U_{3}}{3}\right)$ using the corresponding equations and ASHRAE standard data.

The thermocouple temperatures obtained from the building surface were through an Extech 3-channel data logger, which had conductive probes to measure surface temperatures. These probes were secured to the indoor and outdoor surfaces using electrical tape for average durations of 20-30 s to obtain a steady reading of the surface measured. Different points on the surface were used, and, if the temperature readings did not differ too greatly from one another within that time frame, average values were taken.

According to the results obtained from the thermal images, the single-pane window U-values (Twamley building) were always more than the double-pane window (Museum building) U-values due to the fact that the double-pane windows consist of an extra layer of air that acts as an insulation to the heat flow. It can also be observed that the wall 1 's (in Table 9) $U_{1}$ values are more consistent with the ASHRAE standard while window 1 's $U_{2}$ and $U_{3}$ values in Table 10 (highlighted in green) are more consistent with the ASHRAE standard than $U_{1}$. As there are many factors that influence U-value estimation (please see the following subsection on uncertainty analysis), additional testing needs to be done through rigorous data collection (multiple time frames, precise indoor temperature readings, varied building types, etc.) to come to accurate conclusions. 
Table 8. Performance of K-means and Threshold-Based Clustering.

\begin{tabular}{|c|c|c|c|c|c|c|c|c|c|c|}
\hline \multirow{2}{*}{\multicolumn{2}{|c|}{$\begin{array}{c}\text { Object of Interest } \\
\text { Buildings }\end{array}$}} & \multicolumn{4}{|c|}{ Walls } & \multicolumn{5}{|c|}{ Windows } \\
\hline & & \multicolumn{2}{|c|}{ Museum } & \multicolumn{2}{|c|}{ Twamley } & \multicolumn{2}{|c|}{ Museum } & \multicolumn{2}{|c|}{ Twamley } & \\
\hline \multirow{5}{*}{ Morning } & No. of Pixels (Object) & 197,186 & 197,186 & 186,601 & 186,601 & 36,167 & 366,167 & 17,926 & 17,926 & \multirow{5}{*}{$280.35 \mathrm{~K}$} \\
\hline & Density (Hotspot) & 3.72 & 4.71 & 2.84 & 4.15 & 5.18 & 8.42 & 3.56 & 3.39 & \\
\hline & No. of Pixels (Object) & 7241 & 9266 & 5340 & 8165 & 2022 & 3560 & 4383 & 4098 & \\
\hline & Avg. Temp (Hotspot) & 269.71 & 270.08 & 270.09 & 270.15 & 268.91 & 268.8 & 268.72 & 268.71 & \\
\hline & Avg. Temp (Object) & 268.48 & 268.48 & 268.7 & 268.7 & 268.04 & 268.04 & 268.17 & 268.17 & \\
\hline \multirow{5}{*}{ Evening } & No. of Pixels (Object) & 200325 & 200,325 & 183,642 & 183,642 & 42572 & 42,572 & 24,204 & 24204 & \multirow{5}{*}{$273.25 \mathrm{~K}$} \\
\hline & Density (Hotspot) & 1.89 & 6.25 & 1.19 & 5.15 & 5.43 & 7.37 & 4.31 & 1.48 & \\
\hline & No. of Pixels (Object) & 3340 & 12,526 & 3410 & 9973 & 2626 & 2802 & 999 & 417 & \\
\hline & Avg. Temp (Hotspot) & 277.27 & 276.98 & 279.24 & 278.81 & 274.36 & 274.32 & 273.66 & 274.14 & \\
\hline & Avg. Temp (Object) & 275.48 & 275.48 & 276.54 & 276.54 & 273.31 & 273.31 & 272.21 & 272.21 & \\
\hline \multirow{5}{*}{ Afternoon } & No. of Pixels (Object) & 202409 & 202,409 & 177,711 & 177,711 & 138,911 & 139,811 & 33,185 & 33,185 & \multirow{5}{*}{$276.25 \mathrm{~K}$} \\
\hline & Density (Hotspot) & 2.99 & 5.66 & 1.19 & 5.76 & 4.7 & 6.22 & 2.18 & 7.31 & \\
\hline & No. of Pixels (Object) & 6221 & 11,560 & 2026 & 9984 & 7023 & 9900 & 775 & 1384 & \\
\hline & Avg. Temp (Hotspot) & 294.28 & 294.12 & 308.58 & 321.25 & 281.7 & 281.32 & 319.68 & 317.33 & \\
\hline & Avg. Temp (Object) & 283.9 & 283.9 & 303.46 & 303.46 & 278.86 & 278.86 & 304.2 & 304.2 & \\
\hline
\end{tabular}

Table 9. Museum U-value estimation (morning) on 17 March 2020.

\begin{tabular}{|c|c|c|c|c|c|c|c|c|c|c|c|}
\hline \multirow{3}{*}{ Building Elements } & \multirow{3}{*}{ \# of Images } & \multicolumn{5}{|c|}{ Temperature Analysis } & \multirow{2}{*}{\multicolumn{4}{|c|}{ U-Value Analysis $\left(\mathrm{W} / \mathrm{m}^{2} \cdot \mathrm{K}\right)$}} & \multirow{3}{*}{ ASHRAE } \\
\hline & & \multicolumn{3}{|c|}{ Surface Temperature (K) } & \multirow{2}{*}{$\begin{array}{l}\text { Thermocouple } \\
\text { Temperature }\end{array}$} & \multirow{2}{*}{$\begin{array}{l}\text { External Air } \\
\text { Temperature }\end{array}$} & & & & & \\
\hline & & Max & Min & Avg & & & $U_{1}$ & $U_{2}$ & $U_{3}$ & $U_{c}$ & \\
\hline Window 1 & 19 & 272.39 & 267.36 & 268.22 & \multirow{4}{*}{$268.45 \mathrm{~K}$} & \multirow{4}{*}{$266.15 \mathrm{~K}$} & 0.73 & 1.98 & 1.96 & 1.53 & 1.98 \\
\hline Window (all) & 321 & 277.27 & 266.15 & 268.5 & & & 1.53 & 3.50 & 3.46 & 2.83 & 1.98 \\
\hline Wall 1 & 435 & 278.8 & 265.85 & 268.53 & & & 1.41 & 2.59 & 2.55 & 2.15 & 0.48 \\
\hline Roof & 11 & 269.55 & 266.5 & 267.25 & & & 0.68 & 3.46 & 3.4 & 2.27 & 0.22 \\
\hline
\end{tabular}

Table 10. Twamley U-value estimation (morning), 17 March 2020.

\begin{tabular}{|c|c|c|c|c|c|c|c|c|c|c|c|}
\hline \multirow{3}{*}{ Building Elements } & \multirow{3}{*}{ \# of Images } & \multicolumn{5}{|c|}{ Temperature Analysis (K) } & \multirow{2}{*}{\multicolumn{4}{|c|}{ U-Value Analysis $\left(\mathrm{W} / \mathrm{m}^{2} \cdot \mathrm{K}\right)$}} & \multirow{3}{*}{ ASHRAE } \\
\hline & & \multicolumn{3}{|c|}{ Surface Temperature } & \multirow{2}{*}{$\begin{array}{l}\text { Thermocouple } \\
\text { Temperature }\end{array}$} & \multirow{2}{*}{$\begin{array}{c}\text { Air } \\
\text { Temperature }\end{array}$} & & & & & \\
\hline & & Max & Min & Avg & & & $U_{1}$ & $U_{2}$ & $U_{3}$ & $U_{c}$ & \\
\hline Window 1 & 150 & 271 & 267 & 268.3 & \multirow{4}{*}{$281.05 \mathrm{~K}$} & \multirow{4}{*}{$266.15 \mathrm{~K}$} & 1.13 & 3.52 & 3.46 & 2.66 & 5.39 \\
\hline Windows (all) & 500 & 279.25 & 266.83 & 268.25 & & & 1.36 & 3.46 & 3.46 & 2.78 & 5.39 \\
\hline Wall 1 & 45 & 281 & 266.08 & 269 & & & 1.79 & 2.61 & 2.55 & 2.32 & 0.48 \\
\hline Roof & 11 & 270.3 & 266.7 & 267.4 & & & 0.73 & 3.46 & 3.46 & 2.55 & 0.22 \\
\hline
\end{tabular}

\subsection{Factors Contributing to Uncertainties in Thermal Data Capture and Processing}

The proposed approach consists of three primary layers: (1) the collection of data and instance segmentation using deep learning; (2) clustering and hotspot detection; and (3) U-value estimation. These three layers contribute to the overall uncertainties of the proposed solution. In the following, we discuss each of these points:

1. Uncertainties associated with image capturing include the following:

- Capturing images of surfaces during the daytime should be planned carefully since solar irradiance can skew readings from the imaging apparatus [60]. Sunlight reflecting on external surfaces, such as brick, which is of high emissivity, will radiate more heat than if the surfaces were under shade. We used images obtained before sunrise and after sunset; however, the effects of incident sunlight will still affect the surface for hours after the surface is shaded.

- Surrounding objects, such as metallic surfaces, may reflect high temperatures, leading to inaccurate surface measurements due to reflecting sunlight [61]. We minimized this bias, recognizing that the buildings in these datasets are adjacent to parking lots, which had vehicles with reflective surfaces. These reflections will influence the thermal readings. 
- Heat and humidity are two atmospheric factors that will influence temperature readings [62]. In regions where the temperatures and relative humidity fluctuate quite frequently, measurements must be systematically recorded when there is acceptable consistency in weather patterns for that day or time.

2. Uncertainties with object detection and instance segmentation: Uncertainty in deep learning can be classified mainly into two types: epistemic uncertainty and aleatory uncertainty. Epistemic uncertainty refers to the uncertainty associated with the objects that the model does not know because the training data was not appropriate. This type of uncertainty arises due to gaps in data and knowledge. We limited this type of uncertainty by generating sufficient data as this results in decreasing epistemic uncertainty. The aleatory uncertainty refers to the type of uncertainty rising from the stochasticity of the observations. This second type of uncertainty cannot be mitigated by providing more data to the models. Given the uncertainty in deep learning, the reading of the data associated with U-value calculation is subsequently uncertain, and there will be some variability the readings and the overall U-value estimation. These variabilities are added to other factors discussed in the previous paragraph.

3. Uncertainties with clustering and hotspot detection: The clustering and hotspot detection are directly related to object detection and instance segmentation and uncertainty associated with deep learning will propagate and create uncertainties associated with this part. Apart from these sources of uncertainty, additional sources exist, such as the observations, background knowledge, the induction principle, and the learning algorithm used for this unduction principle.

4. Uncertainties with U-value estimation: The formulas used for U-values are approximations and depend on many factors that are themselves subject to different types of uncertainties, which can result in different measurements.

Please see Table 11 for quantitative reporting of the precision and average deviation when considering U-value estimation. Based on the results from our analysis and due to the high number of sample points for object-wise U-value estimation, unbiased rounding was used to retain one significant digit after the decimal for precision and error, and two significant digits after the decimal for the average deviation. For instance, the wall precision value for Twamley and the error in the wall readings for the Museum were rounded to $15.1 \%$ and $347.9 \%$ from $15.08 \%$ and $347.91 \%$, respectively. For the purposes of our evaluation, we specify the definition of precision according to ISO 3534-1 [63] to be "the closeness of agreement between independent test results obtained under stipulated conditions."

The error was calculated by considering the \% difference between the empirical observations and the true values (ASHRAE) [64]. This can be considered to be a measure of accuracy. Following the standard definition for "true value", the "true value" refers to values obtained by ASHRAE (which may have had systematic or random uncertainties) and not the absolute value for the measurand that is devoid of any contributing or biasing factors. The average deviation $\left(\Delta U_{a v g}\right)$ is calculated using the following formula:

$$
\Delta U_{a v g}=\sum_{i=1}^{3} \frac{\left|U_{i}-U_{c}\right|}{3}
$$

where $U_{i}$ represents the $U$-values 1,2 , and 3 . The precision is calculated using Equation (16)

$$
\text { Precision }=\frac{\Delta U_{a v g}}{U_{c}} \times 100 \%
$$

As can be seen from Table 11, the average deviation for windows was equal to or higher than those of walls for both the buildings. This means that the variation of U-values from their respective average value $\left(U_{c}\right)$ for a given object was lower in the case of walls than windows. We can infer from this table that the U-value measurements for walls were much more similar to one another relative to the windows' U-values. 
Similarly, in terms of accuracy, the U-values obtained for the windows are closer to the true ASHRAE values. These results also confirm an important result: U-values closer to one another may not necessarily indicate higher accuracies as can be seen when the accuracy for walls are considered. Using our methodology for U-value estimation and when considered relative to windows, it can be said that the measured values for walls are more precise (lower precision) but much less accurate (higher errors).

Table 11. The error, precision, and deviation for U-value estimations.

\begin{tabular}{cccccc}
\hline Building & Object of Interest & Error (\%) & Precision (\%) & $\Delta \boldsymbol{U}_{\text {avg }}$ & $\begin{array}{c}\text { ASHRAE } \\
\text { Standard }\end{array}$ \\
\hline \multirow{2}{*}{ Twamley } & Wall & 383.3 & 15.0 & \pm 0.35 & 0.48 \\
& Window & 48.4 & 33.3 & \pm 0.93 & 5.39 \\
\multirow{2}{*}{ Museum } & Wall & 347.9 & 24.5 & \pm 0.53 & 0.48 \\
& Window & 43.0 & 30.6 & \pm 0.87 & 1.98 \\
\hline
\end{tabular}

\section{Conclusions}

Building thermal performance information is crucial to reducing energy consumption and to achieving zero energy buildings. Researchers have proposed many methodologies over the past decades, including statistical approaches, engineering-based methods, and machine learning. These methods present many limitations; therefore, this study aimed to enhance the building thermal performance with a more precise heat loss quantification and to overcome the complexity of engineering methods.

We proposed a novel method using thermal imagery and deep-learning-based instance segmentation combined with analytical methods to compute U-values. We used thermal images captured by SkySkopes to train the machine learning models. The images were obtained during several flight rounds duringearly dawn to avoid any non-desirable reflections and accounted for several variables, such as the angle and distance to walls. The images obtained were annotated and archived using cloud storage. Several classes were defined, such as the facades of buildings, trees, and windows, after which Mask $\mathrm{R}-\mathrm{CNN}$ was trained and tested.

The confusion matrix and AP were computed to evaluate the performance of the machine learning algorithms. The results indicated that the model trained on augmented datasets achieved total average precision values as high as $79 \%$ for facades, $69 \%$ for windows, and $67 \%$ for roofs. The heat loss calculation was also used to quantify the desired values. We proposed clustering and hotspot detection methods to identify the primary regions of heat loss in the facades and windows of the buildings.

Three measures were used to compare the clustering schemes. The overlap metric indicated a $50 \%$ agreement between the methods; however, we explored the average hotspot temperature metric to obtain a definitive conclusion. A maximum difference of 0.48 degrees was observed for the average hotspot temperature metric on surfaces not affected by sunlight and, thus, was effectively used to confirm our results. This information can be leveraged to make appropriate decisions related to building design and maintenance.

The analysis led to the following conclusions: (1) the proposed data driven approach provided an automatic and reliable process for energy audit applications; (2) our results are broadly consistent with the American Society of Heating, Refrigerating, and Airconditioning Engineers building standards; (3) this research generated new information on the dependency of thermal efficiency, which relies on many factors, including the thermal images acquisition process, building geometry, and indoor heating or cooling conditions; and (4) the findings of this research and the quantitative and qualitative uncertainty analyses will provide a significant starting point for discussion and further research in the area of automated processes for energy audit applications. 
Future work will include re-working Mask R-CNN to analyze more than thermal images and with datasets consisting of more balanced classes. Further studies should investigate the possible effects of the building typologies on the meteorological performances of the proposed method.

Author Contributions: Conceptualization, Y.A., N.S., P.R., and D.S.; methodology, Y.A., S.P., and P.R.; software, D.S., Y.A, N.S., P.R., S.P., and A.B.; validation, Y.A., P.R., and S.P.; formal analysis, Y.A, N.S., D.S., S.P., and A.B.; investigation, Y.A. and S.P.; resources, P.R.; data curation, D.S., Y.A., S.P., N.S., and A.B.; writing—original draft preparation, Y.A.; writing—review and editing, N.S., S.P., A.B., and D.S.; visualization, N.S., S.P., and A.B.; supervision, P.R.; project administration, P.R.; funding acquisition, P.R. All authors have read and agreed to the published version of the manuscript.

Funding: The authors would like to acknowledge the support from the North Dakota Department of Commerce Research ND program (Award\# 22166).

Institutional Review Board Statement: Not applicable.

Informed Consent Statement: Not applicable.

Data Availability Statement: Data can be made available on request due to privacy restrictions. The data presented in this study are available on request from the corresponding author. The data are not publicly available due to a binding legal contract with a private firm (registered name mentioned below in "Acknowledgments") which was responsible for acquiring the data.

Acknowledgments: We thank our collaborator Matt Dunlevy (i.e., CEO of SkySkopes Inc., Grand Forks, ND, USA) for his assistance in acquiring the thermal image datasets. Other advisors of the project include David Flynn, Professor, School of Business and Gautham Krishnamoorthy, Associate Professor for Chemical Engineering at UND.

Conflicts of Interest: The authors declare no conflict of interest.

\section{Abbreviations}

The following abbreviations are used in this manuscript:

$\begin{array}{ll}\text { CNN } & \text { Convolutional neural network } \\ \text { IoU } & \text { Intersection over union } \\ \text { ROI } & \text { Region of interest } \\ \text { YOLO } & \text { You Only Look Once } \\ \text { OGI } & \text { Optical gas imaging } \\ \text { FLIR } & \text { Forward looking infrared } \\ H V A C & \text { Heating, ventilation, and air conditioning } \\ R-C N N & \text { Regions based Convolutional neural network } \\ M a s k R-C N N & \text { Region neural network } \\ A P & \text { Average precision } \\ M A P & \text { Mean average precision } \\ T P & \text { True positive } \\ F P & \text { False positive } \\ T N & \text { True negative } \\ F N & \text { False negative } \\ U_{1} & \text { U-value using formula }(1) \\ U_{2} & \text { U-value using formula }(2) \\ U_{3} & \text { U-value using formula }(3) \\ U_{c} & \text { average U-value of } U_{1}, U_{2}, \text { and } U_{3} \\ \epsilon & \text { Wall emissivity } \\ \sigma & \text { Stephen-Boltzmann constant: } 5.6703 \times 10^{-8} \mathrm{~W} \times \mathrm{m}^{-2} \mathrm{~K}^{-4} \\ T_{S} & \text { Surface temperature } \\ \alpha_{c} & \text { convective heat coefficient } \\ T_{a i} & \text { Constant } \\ T_{i n} & \text { Internal wall temperature } \\ & \end{array}$




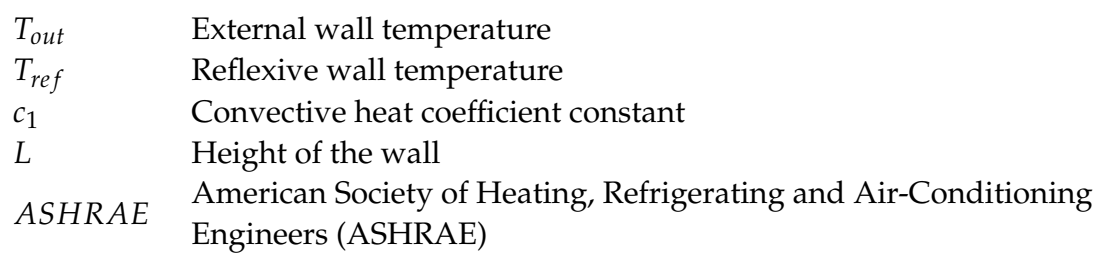

\section{References}

1. Congedo, P.M.; Baglivo, C.; Centonze, G. Walls comparative evaluation for the thermal performance improvement of low-rise residential buildings in warm Mediterranean climate. J. Build. Eng. 2020, 28, 101059. [CrossRef]

2. Bano, F.; Sehgal, V. Evaluation of energy-efficient design strategies: Comparison of the thermal performance of energy-efficient office buildings in composite climate, India. Sol. Energy 2018, 176, 506-519. [CrossRef]

3. Albatici, R.; Tonelli, A.M. Infrared thermovision technique for the assessment of thermal transmittance value of opaque building elements on site. Energy Build. 2010, 42, 2177-2183. [CrossRef]

4. Sadhukhan, D.; Peri, S.; Sugunaraj, N.; Biswas, A.; Selvaraj, D.F.; Koiner, K.; Rosener, A.; Dunlevy, M.; Goveas, N.; Flynn, D.; et al. Estimating surface temperature from thermal imagery of buildings for accurate thermal transmittance (U-value): A machine learning perspective. J. Build. Eng. 2020, 32, 101637. [CrossRef]

5. Lucchi, E. Thermal transmittance of historical stone masonries: A comparison among standard, calculated and measured data. Energy Build. 2017, 151, 393-405. [CrossRef]

6. OǴrady, M.; Lechowska, A.A.; Harte, A.M. Infrared thermography technique as an in situ method of assessing heat loss through thermal bridging. Energy Build. 2017, 135, 20-32. [CrossRef]

7. Wiggenhauser, H. Active IR-applications in civil engineering. Infrared Phys. Technol. 2002, 43, 233-238. [CrossRef]

8. Ali, R.; Zeng, J.; Kavgic, M.; Cha, Y.J. Heat loss detection using thermal imaging by a small UAV prototype. In Smart Structures and NDE for Industry 4.0, Smart Cities, and Energy Systems; International Society for Optics and Photonics: Bellingham, WA, USA, 2020; Volume 11382, p. 113820S.

9. Daffara, C.; Muradore, R.; Piccinelli, N.; Gaburro, N.; de Rubeis, T.; Ambrosini, D. A Cost-Effective System for Aerial 3D Thermography of Buildings. J. Imaging 2020, 6, 76. [CrossRef]

10. Koiner, K.; Rosener, A.; Sadhukhan, D.; Selvaraj, D.F.; Mrabet, Z.E.; Dunlevy, M.; Ranganathan, P. Heat loss estimation using UAS thermal imagery. In Proceedings of the 2019 IEEE International Conference on Electro Information Technology (EIT), Brookings, SD, USA, 20-22 May 2019.

11. Minaee, S.; Boykov, Y.Y.; Porikli, F.; Plaza, A.J.; Kehtarnavaz, N.; Terzopoulos, D. Image Segmentation Using Deep Learning: A Survey. IEEE Trans. Pattern Anal. Mach. Intell. 2021. [CrossRef] [PubMed]

12. Hafiz, A.M.; Bhat, G.M. A survey on instance segmentation: State of the art. Int. J. Multimed. Inf. Retr. 2020, 1-19. [CrossRef]

13. Zhou, R.; Liu, F.; Gravelle, C.W. Deep learning for modulation recognition: A survey with a demonstration. IEEE Access 2020, 8, 67366-67376. [CrossRef]

14. Guo, J.; He, H.; He, T.; Lausen, L.; Li, M.; Lin, H.; Shi, X.; Wang, C.; Xie, J.; Zha, S.; et al. GluonCV and GluonNLP: Deep Learning in Computer Vision and Natural Language Processing. J. Mach. Learn. Res. 2020, 21, 1-7.

15. Arjoune, Y.; Peri, S.; Sugunaraj, N.; Sadhukhan, D.; Nord, M.; Krishnamoorthy, G.; Flynn, D.; Ranganathan, P. Thermal Imagery Based Instance Segmentation for Energy Audit Applications in Buildings. In Proceedings of the 2019 IEEE International Conference on Big Data (Big Data), Los Angeles, CA, USA, 9-12 December 2019; pp. 5974-5976.

16. Szegedy, C.; Vanhoucke, V.; Ioffe, S.; Shlens, J.; Wojna, Z. Rethinking the inception architecture for computer vision. In Proceedings of the IEEE conference on computer vision and pattern recognition, Las Vegas, NV, USA, 27-30 June 2016; pp. 2818-2826.

17. LeCun, Y.; Bengio, Y.; Hinton, G. Deep learning. Nature 2015, 521, 436. [CrossRef]

18. Murugan, V.; Vijaykumar, V.; Nidhila, A. A deep learning RCNN approach for vehicle recognition in traffic surveillance system. In Proceedings of the 2019 International Conference on Communication and Signal Processing (ICCSP), Chennai, India, 4-6 April 2019; pp. 0157-0160.

19. Gupta, A.K. Time portability evaluation of RCNN technique of OD object detection-Machine learning (artificial intelligence). In Proceedings of the 2017 International Conference on Energy, Communication, Data Analytics and Soft Computing (ICECDS), Chennai, India, 1-2 August 2017; pp. 3127-3133.

20. Girshick, R. Fast r-cnn. In Proceedings of the IEEE International Conference on Computer Vision, Santiago, Chile, 7-13 December 2015; pp. 1440-1448.

21. Ren, S.; He, K.; Girshick, R.; Sun, J. Faster r-cnn: Towards real-time object detection with region proposal networks. IEEE Trans. Pattern Anal. Mach. Intell. 2017, 39, 1137-1149. [CrossRef] [PubMed]

22. Tang, J.; Mao, Y.; Wang, J.; Wang, L. Multi-task Enhanced Dam Crack Image Detection Based on Faster R-CNN. In Proceedings of the 2019 IEEE 4th International Conference on Image, Vision and Computing (ICIVC), Xiamen, China, 5-7 July 2019; pp. 336-340.

23. You, W.; Chen, L.; Mo, Z. Soldered Dots Detection of Automobile Door Panels based on Faster R-CNN Model. In Proceedings of the 2019 Chinese Control And Decision Conference (CCDC), Nanchang, China, 3-5 June 2019; pp. 5314-5318.

24. Redmon, J.; Divvala, S.; Girshick, R.; Farhadi, A. You only look once: Unified, real-time object detection. In Proceedings of the IEEE conference on computer vision and pattern recognition, Las Vegas, NV, USA, 27-30 June 2016; pp. 779-788. 
25. Nie, Y.; Sommella, P.; O’Nils, M.; Liguori, C.; Lundgren, J. Automatic Detection of Melanoma with Yolo Deep Convolutional Neural Networks. In Proceedings of the 2019 E-Health and Bioengineering Conference (EHB), Iasi, Romania, 21-23 November 2019; pp. 1-4.

26. Noh, H.; Hong, S.; Han, B. Learning deconvolution network for semantic segmentation. In Proceedings of the IEEE International Conference on Computer Vision, Santiago, Chile, 7-13 December 2015; pp. 1520-1528.

27. Long, J.; Shelhamer, E.; Darrell, T. Fully convolutional networks for semantic segmentation. In Proceedings of the IEEE Conference on Computer Vision and Pattern Recognition, Salt Lake City, UT, USA, 18-23 June 2015; pp. 3431-3440.

28. Liu, W.; Rabinovich, A.; Berg, A.C. Parsenet: Looking wider to see better. arXiv 2015, arXiv:1506.04579.

29. Chen, L.; Papandreou, G.; Kokkinos, I.; Murphy, K.; Yuille, A.L. DeepLab: Semantic Image Segmentation with Deep Convolutional Nets, Atrous Convolution, and Fully Connected CRFs. IEEE Trans. Pattern Anal. Mach. Intell. 2018, 40, 834-848. [CrossRef]

30. Zhang, H.; Dana, K.; Shi, J.; Zhang, Z.; Wang, X.; Tyagi, A.; Agrawal, A. Context encoding for semantic segmentation. In Proceedings of the IEEE Conference on Computer Vision and Pattern Recognition, Salt Lake City, UT, USA, 18-23 June 2018; pp. 7151-7160.

31. Hoang, V.; Hoang, V.; Jo, K. Realtime Multi-Person Pose Estimation with RCNN and Depthwise Separable Convolution. In Proceedings of the 2020 RIVF International Conference on Computing and Communication Technologies (RIVF), Ho Chi Minh City, Vietnam, 14-15 October 2020; pp. 1-5.

32. You, J. Weather Data Integrated Mask R-CNN for Automatic Road Surface Condition Monitoring. In Proceedings of the 2019 IEEE Visual Communications and Image Processing (VCIP), Sydney, Australia, 1-4 December 2019; pp. 1-4.

33. Kirillov, A.; He, K.; Girshick, R.; Rother, C.; Dollár, P. Panoptic segmentation. In Proceedings of the IEEE Conference on Computer Vision and Pattern Recognition, Long Beach, CA, USA, 15-20 June 2019; pp. 9404-9413.

34. Ronneberger, O.; Fischer, P.; Brox, T. U-net: Convolutional networks for biomedical image segmentation. In Proceedings of the International Conference on Medical Image Computing and Computer-Assisted Intervention, Munich, Germany, 5-9 October 2015; Springer: Berlin/Heidelberg, Germany, 2015; pp. 234-241.

35. Chen, L.C.; Papandreou, G.; Schroff, F.; Adam, H. Rethinking atrous convolution for semantic image segmentation. arXiv 2017, arXiv:1706.05587.

36. He, K.; Gkioxari, G.; Dollár, P.; Girshick, R. Mask r-cnn. In Proceedings of the IEEE International Conference on Computer Vision, Venice, Italy, 22-29 October 2017; pp. 2961-2969.

37. Ngo, G.C.; Macabebe, E.Q.B. Image segmentation using K-means color quantization and density-based spatial clustering of applications with noise (DBSCAN) for hotspot detection in photovoltaic modules. In Proceedings of the 2016 IEEE region 10 conference (TENCON), Singapore, 22-25 November 2016; pp. 1614-1618.

38. Hajela, G.; Chawla, M.; Rasool, A. A Clustering Based Hotspot Identification Approach For Crime Prediction. Procedia Comput. Sci. 2020, 167, 1462-1470. [CrossRef]

39. Jaishri, W.; Tamilkodi, R.; Kumari, G.R.N.; Maruthuperumal, S. An innovative method of acquiring optimization for image retrieval via dual clustering method based on segmentation. In Proceedings of the 2016 3rd International Conference on Computing for Sustainable Global Development (INDIACom), New Delhi, India, 16-18 March 2016; pp. 436-440.

40. Kumar, D.; Agrawal, R.K.; Kirar, J.S. Intuitionistic Fuzzy Clustering Method with Spatial Information for MRI Image Segmentation. In Proceedings of the 2019 IEEE International Conference on Fuzzy Systems (FUZZ-IEEE), New Orleans, LA, USA, 23-26 June 2019; pp. 1-7.

41. Naik, P.P.S.; Gopal, T.V. A novel approach for color image segmentation using iterative partitioning mean shift clustering algorithm. In Proceedings of the 2015 International Conference on Communications and Signal Processing (ICCSP), Melmaruvathur, India, 2-4 April 2015; pp. 1516-1519.

42. Melnykov, V.; Michael, S. Clustering large datasets by merging K-means solutions. J. Classif. 2019, 37, 1-27. [CrossRef]

43. Tejedor, B.; Barreira, E.; Almeida, R.M.; Casals, M. Automated data-processing technique: 2D Map for identifying the distribution of the U-value in building elements by quantitative internal thermography. Autom. Constr. 2021, 122, 103478. [CrossRef]

44. Bienvenido-Huertas, D.; Rubio-Bellido, C.; Pérez-Ordóñez, J.L.; Oliveira, M.J. Automation and optimization of in-situ assessment of wall thermal transmittance using a Random Forest algorithm. Build. Environ. 2020, 168, 106479. [CrossRef]

45. Martinez-De Dios, J.; Ollero, A. Automatic detection of windows thermal heat losses in buildings using UAVs. In Proceedings of the 2006 World Automation Congress, Budapest, Hungary, 24-26 July 2006; pp. 1-6.

46. Pedregosa, F.; Varoquaux, G.; Gramfort, A.; Michel, V.; Thirion, B.; Grisel, O.; Blondel, M.; Prettenhofer, P.; Weiss, R.; Dubourg, V.; et al. Scikit-learn: Machine learning in Python. J. Mach. Learn. Res. 2011, 12, 2825-2830.

47. Arthur, D.; Vassilvitskii, S. k-Means++: The Advantages of Careful Seeding; Technical Report; Stanford Infolab: Stanford, CA, USA, 2006.

48. Kodinariya, T.M.; Makwana, P.R. Review on determining number of Cluster in K-Means Clustering. Int. J. 2013, 1, 90-95.

49. Tiihonen, T. Stefan Boltzmann radiation on non convex surfaces. Math. Methods Appl. Sci. 1997, 20, 47-57. [CrossRef]

50. Albatici, R.; Tonelli, A.M.; Chiogna, M. A comprehensive experimental approach for the validation of quantitative infrared thermography in the evaluation of building thermal transmittance. Appl. Energy 2015, 141, 218-228. [CrossRef]

51. Albatici, R.; Passerini, F.; Tonelli, A.M.; Gialanella, S. Assessment of the thermal emissivity value of building materials using an infrared thermovision technique emissometer. Energy Build. 2013, 66, 33-40. [CrossRef] 
52. Nardi, I.; Paoletti, D.; Ambrosini, D.; De Rubeis, T.; Sfarra, S. U value assessment by infrared thermography: A comparison of different calculation methods in a Guarded Hot Box. Energy Build. 2016, 122, 211-221. [CrossRef]

53. Kim, S.J.; Lee, S.W. Air Cooling Technology for Electronic Equipment; CRC Press: Boca Raton, FL, USA, 1996.

54. Fokaides, P.A.; Kalogirou, S.A. Application of infrared thermography for the determination of the overall heat transfer coefficient (U-Value) in building envelopes. Appl. Energy 2011, 88, 4358-4365. [CrossRef]

55. Madding, R. Finding R-values of stud frame constructed houses with IR thermography. Proc. InfraMation 2008, $2008,261-277$.

56. Omega.com. Table of Total Emissivity. Omega. Com 2000, 212, 88-90.

57. Optotherm Thermal Imaging. Emissivity Table for Infrared Thermometer Readings. Available online: https://www.optotherm. com/emiss-table.htm (accessed on 23 May 2021).

58. Rousseeuw, P.J. Silhouettes: A graphical aid to the interpretation and validation of cluster analysis. J. Comput. Appl. Math. 1987, 20, 53-65. [CrossRef]

59. Davies, D.L.; Bouldin, D.W. A Cluster Separation Measure. IEEE Trans. Pattern Anal. Mach. Intell. 1979, PAMI-1, 224-227. [CrossRef]

60. Balaras, C.; Argiriou, A. Infrared thermography for building diagnostics. Energy Build. 2002, 34, 171-183. [CrossRef]

61. Kylili, A.; Fokaides, P.A.; Christou, P.; Kalogirou, S.A. Infrared thermography (IRT) applications for building diagnostics: A review. Appl. Energy 2014, 134, 531-549. [CrossRef]

62. Plesu, R.; Teodoriu, G.; Taranu, G. Infrared thermography applications for building investigation. Bul. Institutului Politeh. Din Lasi. Sect. Constr. Arhit. 2012, 58, 157.

63. NIST. 2021. Available online: https://www.nist.gov/pml/nist-technical-note-1297/nist-tn-1297-appendix-d1-terminology (accessed on 23 May 2021).

64. Raghavendra, N.; Krishnamurthy, L. Engineering Metrology and Measurements; Oxford University Press: Oxford, UK, 2013. 